\title{
Paclitaxel Nano-Delivery Systems: A Comprehensive Review
}

\section{Ping Ma ${ }^{1}$ and Russell J. Mumper ${ }^{1,2 *}$}

${ }^{1}$ Center for Nanotechnology in Drug Delivery, Division of Molecular Pharmaceutics, UNC Eshelman School of Pharmacy, University of North Carolina at Chapel Hill, Chapel Hill, NC 27599, USA

${ }^{2}$ UNC Lineberger Comprehensive Cancer Center, University of North Carolina at Chapel Hill, NC 27599, USA

\begin{abstract}
Paclitaxel is one of the most effective chemotherapeutic drugs ever developed and is active against a broad range of cancers, such as lung, ovarian, and breast cancers. Due to its low water solubility, paclitaxel is formulated in a mixture of Cremophor EL and dehydrated ethanol $(50: 50, \mathrm{v} / \mathrm{v})$ a combination known as Taxol. However, Taxol has some severe side effects related to Cremophor EL and ethanol. Therefore, there is an urgent need for the development of alternative Taxol formulations. The encapsulation of paclitaxel in biodegradable and non-toxic nanodelivery systems can protect the drug from degradation during circulation and in-turn protect the body from toxic side effects of the drug thereby lowering its toxicity, increasing its circulation half-life, exhibiting improved pharmacokinetic profiles, and demonstrating better patient compliance. Also, nanoparticle-based delivery systems can take advantage of the enhanced permeability and retention (EPR) effect for passive tumor targeting, therefore, they are promising carriers to improve the therapeutic index and decrease the side effects of paclitaxel. To date, paclitaxel albumin-bound nanoparticles $\left(\right.$ Abraxane $^{\circledR}$ ) have been approved by the FDA for the treatment of metastatic breast cancer and nonsmall cell lung cancer (NSCLC). In addition, there are a number of novel paclitaxel nanoparticle formulations in clinical trials. In this comprehensive review, several types of developed paclitaxel nano-delivery systems will be covered and discussed, such as polymeric nanoparticles, lipid-based formulations, polymer conjugates, inorganic nanoparticles, carbon nanotubes, nanocrystals, and cyclodextrin nanoparticles.
\end{abstract}

Keywords: Nanoparticles; Poly(lactic-co-glycolic acid); Nanocapsules; Drug-polymer conjugates; Multi-drug resistance; Solid lipid nanoparticles

Abbreviations: Ab: Antibody; Au NPs: Gold Nanoparticles; AUC: Area Under the Curve; BBB: Blood-Brain Barrier; BrC16: 2'-2-Bro-Mohexadecanoyl; Brij 78: Polyoxyl 20-Stearyl Ether; BSA: Bovine Serum Albumin; C22-PX: 2'-Behenoyl-Paclitaxel Conjugate; CD: Cyclodextrin; $\mathrm{CHO}$ : Cholesterol; $\mathrm{C}_{\max }$ : Maximum Concentration; CMC: Critical Micelle Concentration; CNT: Carbon Nanotubes; DHA: Docosahexaenoic Acid; DLPC: 1,2-Dilauroylphosphatidylcholine; DMAB: Didodecyldimethylammonium Bromide; DNA: Deoxyribonucleic Acid; DOPC: 1,2-Dioleoyl-Sn-Glycero-3-Phosphocholine; DOTAP: N-[1[2,3-Dioleoyloxy]Propyl]-N,N,N-Trimethyl-Ammonium Methylsulfate; DPPC: Dipalmitoyl-Phosphatidylcholine; DSPC: 1,2-DistearoylSn-Glycero-3-Phosphocholine; EE: Entrapment Efficiency; EPC: Egg Phosphatidylcholine; EPR: Enhanced Permeability and Retention; FA: Fatty Acid; FITC: Fluorescein Isothiocyanate; h: Hour; HA: Hyaluronic Acid; HER2: Human Epidermal Growth Factor Receptor 2; HO-GC: Hydrotropic Oligomer-Glycol Chitosan; HPG: Hyperbranched Polyglycerol; HPMA: N-[2-Hydroxypropyl]Methacrylamide; HSA: Human Serum Albumin; HSPC: Hydrogenated Soybean Phosphatidylcholine; $\mathrm{IC}_{50}$ : Half Maximal Inhibitory Concentration; i.p: Intraperitoneal; i.v: Intravenous; kg: Kilogram; LRP1: Low-Density Lipoprotein Receptor-Related Protein 1; mAb: Monoclonal Antibody; MDR: Multiple Drug Resistance; mg: Milligram; min: Minute; mL: Milliliter; MMT: Montmorillonite; MNP: Magnetic Nanoparticle; mPEG: Methoxy Poly[Ethylene Glycol]; MTD: Maximum Tolerated Dose; NC: Nanocapsule; NSCLC: Non-Small Cell Lung Cancer; ng: Nanogram; NMR: Nuclear Magnetic Resonance; NP: Nanoparticle; OSA: Octyl-Modified Bovine Serum Albumin; PACA: Poly[Alkyl Cyanoacrylate]; PAMAM: Poly[Amidoamine]; PbAE: Poly[ $\beta$-Amino Ester]; PBCA: Poly[Butyl Cyanoacrylate]; PCL: Poly [ $\varepsilon$-Caprolactone]; PE: Phosphatidyl Ethanolamine; PEEP: Poly[Ethyl Ethylene Phosphate]; PEG: Poly[Ethylene Glycol]; PEG-DSPE: Polyethylene Glycol-Distearoylphosphatidylethanolamine; PEI: Polyethylenimine; PEO-b-PCL: Poly[Ethylene Oxide]block-Poly[ $\varepsilon$-Caprolactone]; PEO-PbAE: Poly[Ethylene Oxide]-Mod- ified Poly[ $\beta$-Amino Ester]; PEO-PPO-PEO: Poly[Ethylene Oxide]Poly[Propylene Oxide]-Poly[Ethylene Oxide]; PEtOz: Poly[2-Ethyl2-Oxazoline]; PG: Poly[L-Glutamic Acid]; PGG: Poly[L- $\gamma$-GlutamylGlutamine]; P-gp: P-glycoprotein; PLA: Poly[L-Lactide]; PLGA: Poly[Lactic-Co-Glycolic Acid]; Pluronic P85: Poly[Oxyethylene-bOxypropylene-b-Oxyethylene]; PPEEA : Poly[2-Aminoethyl Ethylene Phosphate]; PVA: Poly[Vinyl Alcohol]; PX: Paclitaxel; Ref: Reference; RES: Reticuloendothelial System; RHAMM: Hyaluronan-Mediated Motility Receptor; RNA: Ribonucleic Acid; s.c.: Subcutaneous; SD: Standard Deviation; SEC: Size Exclusion Chromatography; siRNA: Small Interfering RNA; SLN: Solid Lipid Nanoparticle; SPAnNa: Poly [Aniline-co-Sodium N-[1-One-Butyric Acid] Aniline]; SSMM: Sterically Stabilized Mixed Micelle; $t_{1 / 2}:$ Half-Life; TEM: Transmission Electron Microscopy; TPGS: D- $\alpha$-Tocopheryl Polyethylene Glycol 1000 Succinate; $\mu \mathrm{g}$ : Microgram; $\mu \mathrm{L}$ : Microliter; VIP : Vasoactive Intestinal Peptide; vs: Versus

\section{Paclitaxel and its Limitations}

Paclitaxel (PX), isolated from the bark of Pacific Yew (Taxus brevifolia), which was first discovered by Mrs. Monroe E. Wall and Mansukh C. Wani, is a white crystalline powder with the melting point

${ }^{*}$ Corresponding author: Russell J. Mumper, Vice Dean and John A. McNeill Distinguished Professor, Center for Nanotechnology in Drug Delivery, Division of Molecular Pharmaceutics, UNC Eshelman School of Pharmacy CB\# 7355, 100G Beard Hall, University of North Carolina at Chapel Hill, Chapel Hill, North Carolina 27599-7355, USA, Tel: +1-919-966-1271; Fax: +1-919-966-6919; E-mail: mumper@email.unc.edu

Received January 17, 2013; Accepted February 15, 2013; Published February 18,2013

Citation: Ma P, Mumper RJ (2013) Paclitaxel Nano-Delivery Systems: A Comprehensive Review. J Nanomed Nanotechol 4: 164. doi:10.4172/21577439.1000164

Copyright: (c) 2013 Ma P, et al. This is an open-access article distributed under the terms of the Creative Commons Attribution License, which permits unrestricted use, distribution, and reproduction in any medium, provided the original author and source are credited. 
of $\sim 210^{\circ} \mathrm{C}$ (Figure 1). It is one of the most effective chemotherapeutic drugs and is mainly used to treat lung, ovarian, and breast cancer, etc [1]. The mechanism of action of PX is to promote and stabilize microtubules and inhibit late $\mathrm{G} 2$ or $\mathrm{M}$ phases of cell cycle, thereby causing the cell death. The major limitation of PX is its low water solubility $(\sim 0.4 \mu \mathrm{g} /$ $\mathrm{mL}$ ); thus, it is formulated in organic solvents of polyoxyethylated castor oil (Cremophor EL) and dehydrated ethanol (50/50, v/v) under the trademark "Taxol". However, Cremophor EL is known to cause serious side effects, such as hypersensitivity reactions [2]. As a result, prolonged infusion time and pretreatments are required. Moreover, the presence of Cremophor EL alters the pharmacokinetic profile of PX in vivo which was described as unpredictable non-linear plasma pharmacokinetics when PX was formulated in Cremophor EL [3]. In addition, PX is a substrate of P-glycoprotein (P-gp), which actively pumps PX out of the cells and induces drug resistance [4]. To overcome this problem, several P-gp inhibitors, such as verapamil [5] and PSC 833 [6], were co-administered with Taxol but the results were disappointing due to their toxicity and/or alteration of PX pharmacokinetics and biodistribution. Nano-delivery systems are promising vehicles in drug delivery because they improve solubility of hydrophobic drugs, such as PX, and generally have low toxicity as well. Abraxane ${ }^{\circledR}$, a PX albuminbound NP formulation with the particle size of $\sim 130 \mathrm{~nm}$, was approved by the FDA in 2005 for the treatment of metastatic breast cancer. This formulation had demonstrated some advantages in terms of reduced toxicity compared to Taxol. In addition, the total dose can be administered within $30 \mathrm{~min}$ without pretreatment. However, whether Abraxane $^{\circledR}$ could improve survival and address P-gp-mediated drug resistance is still unclear. Therefore, the alternative PX formulations are still in demand. In this review, various nanoparticle (NP) systems for the delivery of PX will be addressed, such as polymeric NPs, lipidbased NP formulations, polymer conjugates, inorganic NPs, carbon nanotubes, nanocrystals, cyclodextrin NPs, etc.

\section{Advantages of Nanoparticle-Based Paclitaxel Delivery Systems}

Nanoparticle delivery systems have attracted increasing attention in recent years, especially for cancer therapies. As an effective chemotherapeutic agent, PX has been formulated in various nanodelivery systems which have several advantages over the standard-ofcare therapy. First, the aqueous solubility of PX can be greatly enhanced when it is conjugated with water-soluble polymers, or encapsulated into lipid-based NPs. Second, they are small in size (several to several hundred nanometers in diameter), which enables the preferential delivery of PX into the tumor site due to the enhanced permeability and retention (EPR) effect. Third, they can escape the recognition of reticuloendothelial system (RES) in healthy tissues and therefore reduce the side effects of the drug. As a consequence, higher maximum

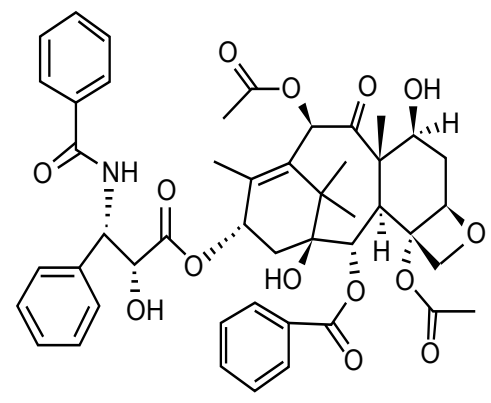

Figure 1: Chemical Structure of $P X$. tolerated doses (MTD) of NPs are realized. It should be noted that, in general, the addition of polyethylene glycol (PEG) on the surface of NPs is required to avoid RES clearance [7]. Fourth, the pharmacokinetic profiles of the drug from NPs is improved, for example, increasing the half-life and tumor accumulation of PX. Last, but not the least, the surface of PX NP systems can be functionalized with active ligands for targeting purpose, which in-turn will further increase the tumor uptake and decrease the side effects of the drug. For more details about the advantages of NP-based drug delivery systems, please refer to the referenced review articles [8-12].

\section{Polymeric Nanoparticles}

A summary of PX-loaded polymeric NPs is shown in Table 1.

\section{Poly (lactic-co-glycolic acid) (PLGA) Nanoparticles}

PLGA is one of the most widely used biodegradable co-polymers for the development of nano-delivery systems because it undergoes hydrolysis in the body and produces non-toxic products of lactic acid and glycolic acid, and eventually carbon dioxide and water. Since the body effectively deals with both degradants, the systemic toxicity associated with PLGA is minimal.

PX-loaded PLGA NPs have been engineered by different methods, such as o/w emulsion-solvent evaporation [13,14], nanoprecipitation [15] and interfacial deposition methods [16]. In most cases, PX was released from PLGA NPs in a biphasic pattern with a fast initial release during the first 1-3 days followed by a slow and continuous release $[13,14,16-18]$. PX-encapsulated PLGA NPs demonstrated enhanced in vitro cytotoxicity as compared to free $\mathrm{PX}$ in various cancer cell lines, such as glioma C6 cells [17], NCI-H69 human small cell lung cancer cells [16], MCF-7 [18] and HeLa cells [15,18]. Furthermore, in vivo PXloaded PLGA NPs showed significantly better tumor growth inhibition effect with transplantable liver tumors [15].

The surface of PLGA NPs was modified for improved drug delivery. Chitosan-coated PLGA NPs exhibited slower in vitro drug release compared to non-coated PLGA NPs and significantly changed the zeta potential from the negative charge of $-30.1 \mathrm{mV}$ for PLGA NPs alone to the positive charge of $26 \mathrm{mV}$, which facilitated drug cell uptake than uncoated NPs [19]. Chakravarthi et al. [20] showed a 4-10-fold increase in cellular association of PX and enhanced cytotoxicity when applied chitosan-modified PLGA NPs. In addition to chitosan, didodecyldimethylammonium bromide (DMAB), a cationic surfactant, was also applied to absorb on the surface of PX-loaded NPs by electrostatic attraction. Upon the addition of DMAB, the negativelycharged NPs shifted to become positively-charged [21]. This DMAB modified PX-incorporated PLGA NPs completely inhibited intimal proliferation in a rabbit vascular injury model [22].

PLGA NPs were also optimized using different emulsifiers. It is known that the employed emulsifiers/stabilizers could have strong influence on the properties of produced NPs, such as morphology, particle size, drug entrapment efficiency, in vitro release behavior, cellular uptake, in vitro cytotoxicity, pharmacokinetics and biodistribution, and as a consequence therapeutic efficacy [23]. Poly (vinyl alcohol) (PVA) is the most commonly used emulsifier. Other emulsifiers were also applied in PLGA NPs. For example, when d- $\alpha$-tocopheryl polyethylene glycol 1000 succinate (TPGS) was utilized in PX-loaded PLGA NPs as the surfactant emulsifier, the PLGA/TPGS NPs could achieve drug encapsulation efficiency of $100 \%$ [24], better controlled drug release kinetics [25], and enhanced cellular uptake and cytotoxicity [26] compared to that of PVA-emulsified PLGA NPs. The TPGS-emulsified 
Citation: Ma P, Mumper RJ (2013) Paclitaxel Nano-Delivery Systems: A Comprehensive Review. J Nanomed Nanotechol 4: 164. doi:10.4172/21577439.1000164

Page 3 of 16

\begin{tabular}{|c|c|c|c|c|c|}
\hline Polymer & Modification & NP Preparation Method & $\% \mathrm{EE}^{*}$ & Status & References \\
\hline \multirow{12}{*}{ PLGA } & - & emulsion-solvent evaporation & 85 & in-vitro & [14] \\
\hline & PLGA, PLGA-PEG, PCL-PEG & nanoprecipitation & 70 & in-vivo & [15] \\
\hline & Poloxamer 188 & interfacial deposition & $>90$ & in-vitro & [16] \\
\hline & TPGS (emulsifier) & emulsion-solvent evaporation & 100 & in-vitro & [24] \\
\hline & DLPC (emulsifier) & emulsion-solvent evaporation & $15-56$ & in-vitro & [28] \\
\hline & DPPC (emulsifier) & emulsion-solvent evaporation & $34-45$ & in-vitro & [29] \\
\hline & chitosan & emulsion-solvent evaporation & $75-79$ & in-vitro & [19] \\
\hline & DMAB & emulsion-solvent evaporation & 47 & in-vivo & [21] \\
\hline & MMT & emulsion-solvent evaporation & $\sim 50$ & in-vitro & {$[30]$} \\
\hline & MMT, HER2 (targeting) & emulsion-solvent evaporation & $\sim 50$ & in-vitro & [31] \\
\hline & RGD (targeting) & emulsion-solvent evaporation & $60-65$ & in-vivo & [32] \\
\hline & Pluronic P85, transferrin (targeting) & nanoprecipitation & $70-76$ & in-vivo & [33] \\
\hline \multirow{12}{*}{ PCL } & PEO-PCL & solvent displacement & $>95$ & in-vivo & {$[63,64]$} \\
\hline & PCL-pluronic F68 & emulsion-solvent evaporation & 84 & in-vivo & [72] \\
\hline & $\begin{array}{l}\text { PCL-pluronic F68, } \\
\text { DMAB }\end{array}$ & modified solvent displacement & $76-88$ & in-vivo & [193] \\
\hline & $\begin{array}{c}\text { mPEG-PCL, } \\
\text { Angiopep (targeting) }\end{array}$ & emulsion and evaporation & 90 & in-vivo & {$[67,68]$} \\
\hline & mPEG-PCL & solid dispersion & 98 & in-vivo & [69] \\
\hline & PVP-b-PCL & modified nanoprecipitation & 85 & in-vivo & [73] \\
\hline & PEG-PCL & co-solvent extraction & - & in-vivo & {$[66]$} \\
\hline & $\begin{array}{c}\text { PEG-PCL, } \\
\text { folic acid (targeting) }\end{array}$ & dialysis & - & in-vitro & [70] \\
\hline & PCL-g-PVA & dialysis & - & in-vitro & [74] \\
\hline & PEtOz-PCL & dialysis & $5-76$ & in-vitro & [75] \\
\hline & PCL-PEEP, galactosamine, (targeting) & dialysis & - & in-vitro & [76] \\
\hline & mPEG-PCL-PPEEA & emulsion-solvent evaporation & $>90$ & in-vivo & {$[77]$} \\
\hline \multirow{5}{*}{ PLA } & PLA-PEG (diblock) & thin film & 65 & in-vivo & [49] \\
\hline & PLA-PEO (star-branch) & solvent evaporation & $6-56$ & in-vitro & [48] \\
\hline & PVA-PEG & solvent evaporation & $20-62$ & in-vitro & {$[50]$} \\
\hline & Poly(y-glutamic acid), galactosamine (targeting) & solvent evaporation & $50-54$ & in-vivo & [51] \\
\hline & PLA-PEG-PLA, PEG-PLA-PEG & solvent evaporation & $14-31$ & in-vivo & {$[52,53]$} \\
\hline \multirow{6}{*}{ Chitosan } & cholanic acid & dialysis & 92 & in-vivo & {$[78,194]$} \\
\hline & oligomer & dialysis & 97 & in-vivo & [79] \\
\hline & glyceryl monooleate & emulsion-solvent evaporation & $98-100$ & in-vitro & {$[80]$} \\
\hline & mPEG, cholesterol & dialysis & 70 & in-vivo & [81] \\
\hline & $\mathrm{N}$-acetyl histidine & - & - & in-vitro & [82] \\
\hline & stearic acid, glutaraldehyde & ultrasonication & $94-99$ & in-vitro & [83] \\
\hline Gelatin & - & desolvation & $>80$ & in-vivo & [109-112] \\
\hline \multirow{2}{*}{ HA } & - & desolvation & 90 & in-vivo & [95] \\
\hline & oligomer & dialysis & - & in-vitro & [96] \\
\hline \multirow{3}{*}{ PBCA } & pluronic F127 & miniemulsion & 80 & in-vitro & [98] \\
\hline & chitosan & dialysis & 90 & in-vivo & [99] \\
\hline & surfactants (dextran 70, cholesterol, PVA, and lecithin) & polymerization & $60-80$ & in-vitro & [100] \\
\hline \multirow{4}{*}{ Albumin } & - & high-pressure homogenization & - & approved & [88] \\
\hline & CREKA and LyP-1, peptides (targeting) & - & - & in-vivo & [91] \\
\hline & folic acid (targeting) & desolvation & 95 & in-vitro & [92] \\
\hline & octaldehyde & dialysis & 90 & in-vitro & [93] \\
\hline HPG & PEG, PEI & solvent evaporation & - & in-vivo & [102] \\
\hline PEG-PE & EPC, solid triglycerides, cationic Lipofectin lipids & solvent evaporation & $\sim 100$ & in-vivo & [103-106] \\
\hline
\end{tabular}

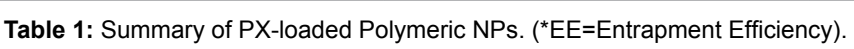

PLGA NPs achieved 10-fold greater bioavailability than Taxol after oral administration [27]. Phospholipids were also used as natural emulsifiers in PLGA NPs, such as 1,2-dilauroylphosphatidylcholine (DLPC) [28] and dipalmitoyl-phosphatidylcholine (DPPC) [29]. Both of the emulsifiers demonstrated greater benefits compared to PVA. Montmorillonite (MMT) was also incorporated into PX-loaded PLGA NPs as both of matrix component and co-emulsifier. The addition of MMT did not change particle size, drug entrapment efficiency, or the in vitro drug release from PLGA NPs. Importantly, the PX-loaded PLGA/
MMT NPs enhanced drug cellular uptake over that of pure PLGA NPs by $57-177 \%$ and $11-55 \%$ in Caco-2 and HT-29 cells, respectively [30]. The PX-loaded PLGA/MMT NPs were further decorated with human epidermal growth factor receptor 2 (HER2) antibodies for targeting purpose, and these targeted NPs exhibited a 12.7-fold enhanced cytotoxicity compared to non-targeted NPs in SK-BR-3 cells [31]. Other targeting ligands, such as RGD [32] and transferrin [33-35], have also been conjugated to PX-encapsulated PLGA NPs for better antitumor efficacy. For example, PX-incorporated PLGA NPs with 
transferrin ligand showed 5-fold enhanced cytotoxicity over that of non-targeted NPs or Taxol. The mice treated with targeted NPs demonstrated complete tumor inhibition and significantly prolonged survival compared to all controls after intratumoral injection in a PC3 prostate cancer mouse model [34].

\section{Poly(lactide) (PLA) Nanoparticles}

PLA is another widely used matrix material for polymeric NP preparation because of its biodegradable and safe properties. Methoxy poly(ethylene glycol)-poly(lactide) co-polymer (mPEG-PLA) was synthesized and incorporated into the NPs to provide long circulating properties. The in vitro cytotoxicity of these NPs increased by 33.3 -fold over that of Taxol after $24 \mathrm{~h}$ in MCF-7 cells. In vivo pharmacokinetic studies demonstrated the AUC and half-life of PX mPEG-PLA NPs in rat plasma were 3.1 - and 2.8 -fold greater than that of Taxol, respectively [36,37]. PX-loaded NPs with PLA and mPEG-PLA at various ratios of $100 / 0,75 / 25,50 / 50,25 / 75$, and $0 / 100$ were evaluated. It was found that as the mPEG-PLA component in the blend increased, the particle size of NPs and the glass transition temperature of PLA decreased, while the zeta potential of NPs and in vitro drug release increased [38]. Copolymers of PLA/Tween 80 were synthesized and PX-loaded PLA/ Tween 80 NPs were shown to be about 3-fold more toxic than PXloaded PLGA NPs in glioma C6 cells [39]. TPGS was also utilized as an emulsifier in PLA NPs. The Feng group [40] synthesized PLA-TPGS co-polymers using a ring-opening polymerization method. Compared to PX-loaded NPs, the PLA/TPGS NPs showed 1.8- and 1.4-fold enhanced cellular uptake of PX in HT-29 and Caco-2 cells, respectively. The $\mathrm{IC}_{50}$ value of PLA/TPGS NPs was also found to be $40 \%$ lower than that of Taxol in HT-29 cells [41]. In vivo this PX-loaded PLA/TPGS NP formulation achieved a 27.4- and 1.6-fold greater half-life and AUC, respectively, in a xenograft tumor model when compared to Taxol [42]. PX-loaded PLA/TPGS NPs with various ratios of PLA and TPGS were evaluated, and the results demonstrated that the PLA/TPGS ratio had little effect on particle size. However, PLA/TPGS NPs with PLA/ TPGS ratio of $89 / 11$ were the optimized formulation in terms of drug entrapment efficiency, cellular uptake, and in vitro cytotoxicity [43]. Folate-decorated PX-loaded PLA-TPGS NPs were further formulated to achieve even better therapeutic effect $[44,45]$. Other targeted PXloaded PLA NPs, such as HER2 [46], biotin and folic acid [47], were also reported to greatly improve efficacy both in vitro and in vivo.

PLA co-polymer micelles have also been reported for PX delivery [48-53]. For example, PX-loaded PEG-b-PLA micelles were prepared and the mechanism of action was investigated. It was found that the micelles first interacted with cell membranes and then the loaded PX was released. After that, PX was internalized into the cells by lipid/ $\mathrm{raft} /$ caveolae-mediated endocytosis pathway. In this way, PEG-bPLA micelles were able to overcome multiple drug resistance (MDR) which was confirmed by the increased cellular uptake of PX in resistant A2780/T cells. The results also suggested PEG-b-PLA micelles could inhibit P-gp efflux [49]. Paxceed ${ }^{\circledR}$ is a polymeric micelle formulation where PX is encapsulated in PLA-b-mPEG diblock co-polymers. The micellar formulation was found to be more efficacious than Taxol at the maximum tolerated dose (MTD) upon intraperitoneal injection in an MV-522 lung tumor bearing mouse model [54]. Currently, Paxceed $^{\circledR}$ is in phase II clinical trials [55]. Genexol-PM remains the most successful PX micellar formulation to date, which is composed of PLA-b-PEG diblock co-polymers [56]. A preclinical in vivo study with Genexol-PM was found to have 3-fold increased MTD and 2-3fold higher drug concentration in various tissues and more importantly in tumors, compared to Taxol in nude mice. The in vivo antitumor efficacy of Genexol-PM was also significantly improved [57]. In phase I clinical studies, the MTD dose was determined to be $180 \mathrm{mg} / \mathrm{m}^{2}$. The plasma AUC and $\mathrm{C}_{\max }$ increased by 3 - and 4 -fold, respectively, when the dose increased from 80 to $200 \mathrm{mg} / \mathrm{m}^{2}$ [58], which suggested the pharmacokinetics of Genexol-PM were dose-proportional. In phase II clinical studies, Genexol-PM was found to be safe and effective in patients with metastatic breast or advanced pancreatic cancer $[59,60]$. Phase III clinical studies are currently in process.

Triblock co-polymers of PLA-PEG-PLA and PEG-PLA-PEG were synthesized as carriers for PX. The results demonstrated that the drug release from PEG-PLA-PEG micelles was slower than from PLA-PEG-PLA micelles, and PEG contents in micelles influenced the stealth properties of the micelles. Both of micelles showed 4 -fold decreased monocyte cell uptake compared to PLA micelles [52,53]. In another study, a four-armed (star-branched) co-polymer of PLA and PEO was synthesized. Compared to di- and tri-block co-polymers, the star-branched micelles exhibited better controlled and more complete release manner over 2 weeks. Furthermore, the star-shaped micelles had smaller particle size which had the potential to take more advantages of the EPR effect in cancer therapy [48].

In addition to PEG-modified PLGA micelles, PX-incorporated PVP-b-PLA micelles were prepared by Gaucher et al. [50] by an o/w emulsion solvent evaporation method. The cryoprotectant property of PVP allowed the same particle size upon reconstitution after lyophilization, while PEG-modified PEG-b-PLA micelles did not. For targeting purpose, a galactosamine targeted PX-loaded micelle formulation composed of poly $(\gamma$-glutamic acid) and PLA was developed. The targeted NP formulation showed the most significant antitumor efficacy compared to other controls and importantly more drug accumulation in tumors was observed in hepatoma tumorbearing nude mice [51].

\section{Poly( $\varepsilon$-caprolactone) (PCL) Nanoparticles}

Deshpande et al. [61] developed poly(ethylene oxide)-modified poly( $\varepsilon$-caprolactone) (PEO-PCL) NPs for co-delivery of PX and $\mathrm{C}_{6}$ ceramide (an apoptotic signaling molecule) to overcome MDR. The prepared PEO-PCL NPs had high drug entrapment efficiency of $>95 \%$ with $\mathrm{PX}$ and $\mathrm{C}_{6}$-ceramide drug loading of $10 \%(\mathrm{w} / \mathrm{w})$. The particle size of the NPs was $\sim 270 \mathrm{~nm}$ in diameter. In resistant human ovarian cancer $\mathrm{SKOV}_{\mathrm{TR}}$ cells, PX and $\mathrm{C}_{6}$-ceramide loaded PEO-PCL NPs showed 100 -fold enhanced cytotoxicity compared to free PX [62]. In vivo PEOPCL NPs demonstrated remarkable tumor growth inhibition in both wild-type SKOV3 and resistant SKOV3 $3_{\mathrm{TR}}$ xenograft mouse models compared to all the controls. The results indicated the combination of $\mathrm{PX}$ and $\mathrm{C}_{6}$-ceramide incorporated into PEO-PCL NPs overcame MDR in ovarian cancer [63]. The combination of PX and tamoxifen loaded PEO-PCL NPs was also evaluated both in vitro and in vivo. In vitro this formulation lowered the $\mathrm{IC}_{50}$ by 10 - and 3 -fold in SKOV3 and SKOV3 cells, respectively, when compared to free $\mathrm{PX}$. The in vivo PEO-PCL NPs significantly enhanced antitumor efficacy and no acute toxicity was observed [64]. Later, polymeric NP systems for the co-delivery of both PX and P-gp silencing siRNA were developed. In order to do that, poly(ethylene oxide)-modified poly $(\beta$-amino ester) (PEO-PbAE) and PEO-PCL NPs were formulated to encapsulate P-gp silencing siRNA and PX, respectively. The co-administration of P-gp silencing siRNAloaded PEO-PbAE NPs and PX-loaded PEO-PCL NPs completely reversed the MDR based on the fact that the similar cytotoxic activity of NPs in both sensitive SKOV3 and resistant SKOV $3_{\text {TR }}$ cells [65].

Similarly, PEG-PCL polymeric micelles were prepared by a co- 
solvent extraction technique. A series of PX prodrugs were synthesized and encapsulated into micelles and it turned out that 7'-hexonoate PX loaded PEG-PCL NPs demonstrated improved pharmacokinetic profile compared to Taxol [66]. Xin et al. [67] prepared mPEG-PCL NPs to encapsulate PX by an emulsion-solvent evaporation method. In vitro PX-loaded mPEG-PCL NPs showed higher cytotoxicity than Taxol and PCL NPs. Importantly, mPEG-PCL NPs increased the mean survival time to 28 days versus 20 and 23 days for Taxol and PCL NPs, respectively, in a C6 tumor-bearing mouse model. Angiopep, a peptide, was further conjugated on mPEG-PCL NPs to target the low-density lipoprotein receptor-related proteins over-expressed on blood-brain barrier (BBB) and facilitate chemotherapeutic agent of PX across BBB [68]. Similarly, Wang and co-workers [69] developed PX-loaded mPEG-PCL NPs by solid dispersion method without the use of organic solvents. The NPs achieved drug loading of $25.6 \%(\mathrm{w} / \mathrm{w})$ and an entrapment efficiency of $98 \%$. The MTD of NPs was 2.6-fold greater than Taxol in BALB/c mice after bolus intravenous injection. The tissue distribution of PX in NPs was different from that of Taxol and significantly greater tumor accumulation was observed. Chen et al. [70] prepared more complicated PEG-PCL NPs where a PEG-PCL diblock co-polymer was hyperbranched aliphatic polyester Boltorn $\mathrm{H} 40$, and then folic acid was further coated on the surface of the NPs. The PX-loaded NPs demonstrated increased cytotoxicity in vitro.

In addition to PEG-modified PCL NPs, a PX-loaded PCL/ pluronic F68 NP (PCL/F68) formulation was prepared using a solvent evaporation method. The incorporation of pluronic F68 into PCL NPs formed pores and thus expedited drug release from NPs. The PX-loaded PCL/F68 NPs had increased cellular uptake and enhanced toxicity as compared to PCL NPs and Taxol in resistant MCF-7/TAX cells [71]. In vivo the PCL/F68 NPs demonstrated the improved tumor inhibitory activity after a single intratumoral injection compared to multiple intraperitoneal injections of Taxol in a murine breast cancer model [72]. DMAB was further adsorbed on PCL/ F68 NPs via chargecharge interaction to enhance NPs internalized into the arterial wall in animal angioplasty models [22].

Other PX-loaded PCL-based NPs have also been reported. For example, PVP-b-PCL NPs demonstrated significantly superior antitumor efficacy to Taxol in hepatic H22 tumor-bearing mice [73]. PCL grafted PVA (PCL-g-PVA) NPs were engineered and PX-loaded PCL-g-PVA NPs were characterized and exhibited reduced drug release rate profiles [74]. PX-loaded poly(2-ethyl-2-oxazoline) (PEtOz)-PCL NPs [75], galactosamine targeted poly(ethyl ethylene phosphate) (PEEP)-PCL NPs [76], and mPEG-PCL-poly(2-aminoethyl ethylene phosphate) (mPEG-PCL-PPEEA) NPs [77] were reported to have better antitumor activity than Taxol in vitro and/or in vivo.

\section{Chitosan Nanoparticles}

Kim et al. [78] synthesized PX-loaded cholanic acid-modified chitosan NPs using a simple dialysis method with drug loading of $10 \%$ $(w / w)$. This NP formulation showed faster cellular uptake and better therapeutic efficacy in SCC7 tumor-bearing mice compared to Taxol. Hydrotropic oligomer-glycol chitosan (HO-GC) was synthesized to enhance the aqueous solubility of PX in NPs, and achieved a higher drug loading up to $20 \%$ and maximum entrapment efficiency of $97 \%$. The PX-encapsulated HO-GC NPs demonstrated a sustained in vitro release profile, and importantly PX predominantly accumulated in tumor site in vivo [79]. Glyceryl monooleate-modified chitosan NPs loaded with PX was reported to increase by 4 - and 1,000-fold the cellular uptake and cytotoxicity, respectively, in MDA-MB-231 cells as compared to free PX [80]. A more complex chitosan derivative modified with mPEG and cholesterol (CHO) was synthesized for PX delivery. The mPEG-CHO-chitosan NPs showed similar cytotoxicity as free PX in vitro, but significantly slower tumor growth rate and improved life span compared to free PX at the dose of $10 \mathrm{mg} / \mathrm{kg}$ in a tumor-bearing mouse model [81]. A pH-sensitive self-assembled modified chitosan NP formulation was developed based on the fact that hydrophobic acetyl histidine modified chitosan could self-assemble NPs at neutral $\mathrm{pH}$ but dissemble at slightly acidic environment due to the protonation of its imidazole group. The PX-loaded modified NPs were then confirmed to endocytose into cells and release drug in acidic endosomes [82]. In another study, the hydrophobic fatty acid of stearic acid was grafted to chitosan oligosaccharide and the micelles were self-assembled. Glutaraldehyde was then crosslinked to the shells of NPs to further increase the stability of the micelles. The PX-loaded glutaraldehyde-crosslinked chitosan oligosaccharide micelles were found to enhance PX solubility, and had less burst release and slower drug release compared to non-crosslinked NPs [83].

\section{Albumin Nanoparticles}

Albumin is a versatile natural protein carrier for targeted drug delivery. Human serum albumin (HSA) is the most abundant plasma protein in the human blood with a half-life of 19 days. It can reversibly bind hydrophobic drug substances, transport them in the body and release drugs at cell surface [84]. In addition, albumin is actively transported into tumors via a selective over-expression of a $60 \mathrm{kDa}$ glycoprotein (gp60) receptor (albondin) [85]. Albumin-bound PX NPs (Abraxane $^{\circledR}$ ) was approved by US FDA in 2005 for the treatment of metastatic breast cancer in patients who fail other chemotherapy or relapse. In October 2012, the FDA approved Abraxane ${ }^{\circledR}$ to treat nonsmall cell lung cancer (NSCLC), the most common form of lung cancer [86]. The albumin-bound PX NPs have an average particle size of about $130 \mathrm{~nm}$. Since this NP formulation completely eliminates Cremophor EL and ethanol in Taxol, it is administered in a shorter period of $30 \mathrm{~min}$ with no need for premedication. For more details about Abraxane ${ }^{\mathbb{R}}$ product, please refer to the review articles [87-90]. Interestingly, targeted Abraxane formulations with two peptides of CREKA and LyP1 (CGNKRTRGC), respectively, were developed and LyP-1-Abraxane demonstrated significantly improved antitumor efficacy compared to untargeted Abraxane in an MDA-MB-435 xenograft mouse model [91]. In addition to human albumin, Zhao et al. [92] prepared PXloaded bovine serum albumin (BSA) NPs using a desolvation method, and subsequently coated NPs by folic acid for targeting. The folatedecorated NPs exhibited high stability and desired surface properties which specifically targeted to human prostate cancer PC3 cells. In another study, a novel octyl-modified bovine serum albumin (OSA) was synthesized to improve the lipophilicity of albumin and facilitate to form PX-loaded core-shell nanomicelles. The OSA NPs had smaller particle size, higher drug entrapment efficiency, and greater stability compared to unmodified NPs [93].

\section{Hyaluronic Acid (HA) Nanoparticles}

HA is a natural non-toxic and biodegradable polysaccharide. Recently, it has been widely used as a targeting agent since most malignant solid tumors over-express HA receptors, such as CD44 and hyaluronan-mediated motility receptor (RHAMM) [94]. PXloaded HA NPs were reported by Al-Ghananeem et al. [95]. Although the in vitro cytotoxicity of NPs was comparable to free PX in both MDA-MB-231 and ZR-75-1 breast cancer cells, PX NPs showed superior antitumor efficacy to free PX when intratumorally injected into 7,12-dimethylbenz[a]anthracene-induced breast cancer tumorbearing rats. A modified HA was synthesized with an amine-terminated 
hydrotropic oligomer, which enhanced PX solubility and achieved the drug loading up to $20.7 \%$. The NPs were found to selectively target SCC-7 cells which over-expressed CD44, and exhibited enhanced cytotoxicity in this cell line than normal fibroblast CV-1 cells [96].

\section{Poly(butyl cyanoacrylate) (PBCA) Nanoparticles}

Biodegradable poly-(alkyl cyanoacrylate) (PACA) NPs have been widely used for the delivery of various active pharmaceutical ingredients during recent 10 years. Among all different kinds of PACA NPs, poly-(butyl cyanoacrylate) (PBCA) is the most widely used as a carrier because it interacts with a variety of drugs [97]. Huang et al. [98] developed PX-loaded PBCA NPs with the addition of pluronic F127 as the surfactant using a mini-emulsion method. It was found that the drug loading and entrapment efficiency of pluronic F127 modified NPs were significantly increased. The PBCA NPs prepared from the mini-emulsions showed a gradual drug release up to $80 \%$ within $96 \mathrm{~h}$. Hyaluronic acid (HA)-coated PBCA NPs encapsulated with PX were reported to reduce the initial burst release of the drug and decrease the cytotoxicity of placebo HA-PBCA NPs compared to non-HA coated PBCA NPs. Importantly, PX-loaded HA-PBCA NPs enhanced by 9.5 -fold the cellular uptake in Sarcoma- 180 cells and showed more potent antitumor inhibition activity versus non-HA coated PX PBCA NPs when injected i.v. into Sarcoma-180 tumor-bearing mice [99]. Mitra and Lin [100] studied the effect of various surfactants on PXencapsulated PBCA NPs. The surfactants of dextran 70, cholesterol, PVA, and lecithin were utilized and the results demonstrated that the NPs incorporated with surfactants were better than without surfactants in terms of particle size and stability. Among the four surfactants, the natural lipids of cholesterol and lecithin were superior to the other two in terms of producing NPs with smaller particle size, higher zeta potential and drug entrapment efficiency, and better controlled drug release profiles.

\section{Hyperbranched Polyglycerol (HPG) Nanoparticles}

HPG is a water-soluble polymer with minimal toxicity. It is one of a few hyperbranched polymers that can be synthesized in a controlled manner [101]. Mugabe et al. [102] loaded PX into two types of modified HPG NPs (HPG-C10-PEG and PEI-C18-HPG) using a solvent evaporation method. The particle size of both NPs was less than $20 \mathrm{~nm}$. The PX was released up to $80 \%$ from HPG-C10-PEG while only 40\% from PEI-C18-HPG. PX-loaded HPG-C10-PEG NP formulation was better tolerated and exhibited significantly improved antitumor efficacy in vivo as compared to Taxol, although the in vitro cytotoxicity was slightly decreased compared to Taxol.

\section{Poly (Ethylene Glycol)-Phosphatidyl Ethanolamine (PEG- PE) Nanoparticles}

The Torchilin group $[103,104]$ developed polymeric micelles composed of PEG and diacyllipids, such as phosphatidyl ethanolamine (PE) for PX delivery. The presence of two hydrocarbon chains of PE increased hydrophobic interactions and therefore provided better stability of the micelles. The PX-loaded micelles had similar size to the placebo micelles. An immunomicelle formulation was prepared by attaching monoclonal antibody $(\mathrm{mAb}) 2 \mathrm{C} 5$ on the surface of the micelles, and it showed better antitumor activity both in vitro and in vivo compared to Taxol or non-targeted micelles in a Lewis lung carcinoma mouse model. Later, PX-loaded mixed micelles were developed by the mixture of PEG-PE and egg phosphatidylcholine, or the mixture of PEG-PE, solid triglycerides, and cationic Lipofectin lipids. The mixed polymeric micelles further improved antitumor efficacy $[105,106]$. The
Rubinstein laboratory prepared sterically stabilized mixed micelles (SSMM) consisting of polyethylene glycol-distearoylphosphatidyleth anolamine (PEG-DSPE) and egg phosphatidylcholine. PX loading in SSMM was 1.5-fold higher than that of PEG-DSPE micelles at the same lipid concentration. PX-loaded SSMM, PEG-DSPE micelles, and free PX had comparable cytotoxicity in MCF-7 cells [107]. Actively targeted vasoactive intestinal peptide (VIP)-grafted SSMM (SSMM-VIP) was further engineered for the targeting purpose. The PX-loaded SSMMVIP was significantly more potent than PX-loaded SSMM and free PX in drug resistant BC19/3 cells [108].

\section{Gelatin Nanoparticles}

Lu et al. [109-112] developed PX-loaded gelatin NPs using a desolvation method. The $\mathrm{IC}_{50}$ value of NPs was nearly identical to that of free PX in RT4 bladder transitional cancer cells. The pharmacokinetic and biodistribution studies indicated that the NP formulation altered drug plasma concentration, clearance and tissue distribution in mice. The PX-loaded gelatin NPs showed significantly improved antitumor activity compared to free PX in intravesical bladder cancer therapy in dogs.

\section{PEG-Polyaspartate Micellar Nanoparticles (NK 105)}

PEG-polyaspartate micellar NPs (NK 105) are constructed by copolymers of PEG and polyaspartate followed by the incorporation of PX into the core of the micelles via hydrophobic interaction [113]. The drug loading was up to $23 \%(\mathrm{w} / \mathrm{w})$ with the particle size of $85 \mathrm{~nm}$. In preclinical studies, NK 105 exhibited 90- and 25-fold greater plasma and tumor AUCs compared to free PX, respectively, and significantly better antitumor efficacy than free PX in a human colorectal cancer HT-29 xenograft mouse model. In addition, the neurotoxicity of NK 105 was dramatically lower than that of free PX [114]. In phase I clinical studies, the MTD of NK 105 was determined to be $180 \mathrm{mg} / \mathrm{m}^{2}$ and the plasma AUC was 15-fold higher than Taxol at the dose of $150 \mathrm{mg} / \mathrm{m}^{2}$ [115]. Currently, NK 105 is in phase III clinical studies.

\section{Lipid-Based Nanoparticles}

A summary of PX-loaded lipid-based NPs is shown in Table 2.

\section{Liposomes}

Liposomes are a mature and versatile drug delivery platform for both lipophilic and hydrophilic compounds. Zhang et al. [116] developed a novel lyophilized liposome-based PX formulation using phosphatidylcholine, cholesterol, and cardiolipin. The particle size did not change upon reconstitution after lyophilization. In addition, the formulation was physically and chemically stable at either $2-8^{\circ} \mathrm{C}$ or $25^{\circ} \mathrm{C}$ for at least 12 months. The in vitro drug release from liposome formulations was slow with less than $6 \%$ of PX released after $120 \mathrm{~h}$. Yoshizawa and co-workers [117] formulated PX into liposomes composed of hydrogenated soybean phosphatidylcholine (HSPC), cholesterol, and PEG-DSPE ${ }_{2000^{\circ}}$ The plasma AUC of PEGylated liposomes was 3.6-fold higher than non-PEGylated liposome formulation in normal rats. In colon-26 solid tumor-bearing mice, the PX-PEGylated liposome had significantly better antitumor efficacy and greater tumor uptake of PX compared to non-PEGylated liposomes. PX-loaded multilamellar liposomes were developed by Fetterly and Straubinger [118] which were composed of phosphatidylglycerol and phosphatidylcholine. Pharmacokinetic and biodistribution studies of PX liposomes were performed. The results showed that the AUC values in blood were comparable to Taxol, but tissue biodistribution was different. In general, the AUCs were significantly higher for Taxol 


\begin{tabular}{|c|c|c|c|c|c|}
\hline Platform & Composition & $\% \mathrm{EE}^{*}$ & Particle Size (nm) & Status & Ref. \\
\hline \multirow{3}{*}{ Liposomes } & phosphatidylcholine, cholesterol, cardiolipin & $>90$ & $\sim 150$ & in-vitro & [116] \\
\hline & HSPC, cholesterol, PEG-DSPE & - & $\sim 100$ & in-vivo & [117] \\
\hline & $\begin{array}{l}\text { phosphatidylglycerol, } \\
\text { phosphatidylcholine }\end{array}$ & - & 200,900 & in-vivo & [118] \\
\hline \multirow{9}{*}{$\begin{array}{l}\text { Solid Lipid } \\
\text { Nanoparticles }\end{array}$} & $\begin{array}{l}\text { tripalmitin, Epikuron 20, butanol, } \\
\text { Na-taurocholate, cholesteryl hemisuccinate }\end{array}$ & - & 160 & in-vitro & [121] \\
\hline & $\begin{array}{l}\text { stearic acid, lecithin, Brij } 78 \\
\text { (or pluronic F68 and DSPE-PEG) }\end{array}$ & $58-75$ & $100-220$ & in-vivo & {$[122]$} \\
\hline & trimyristin, $\mathrm{EPC}, \mathrm{PEG}_{2000}-\mathrm{PE}$ & $72-89$ & $200-250$ & in-vivo & {$[123,124]$} \\
\hline & glyceryl palmitostearate (or glyceryl monostearate), poloxamer 407 & $55-90$ & $\sim 200$ & in-vitro & {$[127,128]$} \\
\hline & $\begin{array}{l}\text { Lipoid S } 100 \text {, } \\
\text { sucrose fatty acid esters }\end{array}$ & $89-92$ & $150-190$ & in-vitro & [129] \\
\hline & stearylamine, soya lecithin, poloxamer 188 & 75 & $70-130$ & in-vivo & [125] \\
\hline & $\begin{array}{l}\text { monostearin, stearic acid, } \\
\text { glycerol tristearate, ATO } 888\end{array}$ & $36-76$ & $160-260$ & in-vitro & {$[126]$} \\
\hline & emulsifying wax, Brij 78 & $\sim 50$ & $<100$ & in-vivo & {$[130,131]$} \\
\hline & glyceryl tridodecanoate, Brij 78 & $>85$ & 170 & in-vitro & {$[132,133]$} \\
\hline \multirow{6}{*}{ Lipid Nanocapsules } & $\begin{array}{l}\text { Labrafac }^{\circledR}, \text { Lipoïd }^{\circledR} \text { S75-3, } \\
\text { Solutol }{ }^{\circledR} \text { HS15 }\end{array}$ & - & $\sim 50$ & in-vivo & {$[134-136]$} \\
\hline & Captex $^{\circledR} 8000$, Lipoïd ${ }^{\circledR}$ S75-3, Solutol ${ }^{\circledR}$ HS15 & 93 & $\sim 50$ & in-vivo & {$[137-140]$} \\
\hline & Miglyol 812, Brij 78, TPGS & $85-97$ & $\sim 200$ & in-vivo & {$[132,133]$} \\
\hline & PLGA, lecithin, DSPE-PEG ${ }_{2000}$, DSPC, RGD (targeting) & 82 & 68 & in-vivo & {$[142,143]$} \\
\hline & $\begin{array}{l}\text { Lipiodol, PEO-PPO-PEO, functionalized PEG, } \\
\text { folic acid (targeting) }\end{array}$ & 46 & 110 & in-vivo & [144] \\
\hline & $\begin{array}{l}\text { lecithin, Dynasan 118, Miglyol 812, folate-PEG-PE, Span } 60 \text { (or PEG- } \\
\text { PE) }\end{array}$ & 98 & 207 & in-vivo & {$[145]$} \\
\hline \multirow{5}{*}{$\begin{array}{l}\text { Micro- and Nano- } \\
\text { Emulsions }\end{array}$} & vitamin E, TPGS, poloxamer 407 & - & 100 & phase III / discontinued & [154] \\
\hline & oil blend, EPC, Tween 80, glycerol & $>95$ & 150 & in-vivo & [156] \\
\hline & $\begin{array}{l}\text { lecithin, butanol, myvacet } \\
\text { (or capmul, myvacet) }\end{array}$ & - & $\sim 110$ & in-vivo & {$[157,158]$} \\
\hline & pine nut oil, egg lecithin, stearylamine, deoxycholic acid & - & $90-120$ & in-vivo & [159] \\
\hline & egg lecithin, flaxseed oil, DSPE-PEG ${ }_{2000}$ & $97-100$ & $130-150$ & in-vitro & {$[160]$} \\
\hline
\end{tabular}

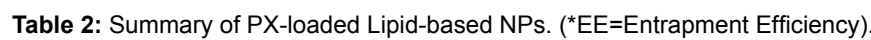

in bone marrow, skin, kidney, brain, adipose, and muscles, indicating the reduced toxicity of liposome formulations.

EndoTAG-1, PX-loaded cationic liposome formulations developed by MediGene, had the potential to treat cancer. EndoTAG-1 consisted of N-(1-(2,3-dioleoyloxy)propyl)-N,N,N-trimethylammonium methylsulfate (DOTAP) and 1,2-dioleoyl-sn-glycero3-phosphocholine (DOPC), which bound to negatively charged endothelial cells in tumor blood vessels. In a prostate cancer mouse model, the mice treated with EndoTAG-1 had significantly smaller tumor volumes compared to the other controls [119]. In a different orthotopic pancreatic cancer model, the combination of EndoTAG-1 and gemcitabine inhibited the pancreatic metastasis [120]. Currently, EndoTAG-1 is in phase II clinical trials.

\section{Solid Lipid Nanoparticles (SLNs)}

Serpe etal. [121] developed PX-loaded SLNs composed of tripalmitin as the oil phase, Epikuron 20 as the surfactant, and Na-taurocholate, butanol, and cholesteryl hemisuccinate as the co-surfactants. Although the in vitro cytotoxicity of the SLNs was comparable to that of Taxol in HT-29 colorectal cancer cells, the combination of PX and doxorubicin (DOX) loaded SLNs exerted a synergistic effect which was more potent than the mixture of Taxol and free DOX. Chen et al. [122] prepared two types of SLNs using Brij 78, or pluronic F68 and PEG-DSPE as the surfactants. Both of PX-incorporated SLNs were proved to have prolonged circulation compared to Taxol in KM mice. Lee and co-workers $[123,124]$ prepared PX-loaded SLNs with the oil phase of trimyristin using a hot homogenization method. The in vitro cytotoxicity of NPs was similar to Taxol in both OVCAR-3 and MCF-7 cells. In vivo pharmacokinetic and biodistribution studies demonstrated the SLN formulation provided the comparable circulation when compared to Taxol after intravenous injection in Sprague-Dawley rats. Pandita et al. [125] prepared PX-encapsulated SLNs using a modified solvent injection method with stearylamine as the lipid. After oral administration of NPs in mice, the AUCs increased by 10- and 2-fold in plasma and most tissues, respectively, as compared to free PX solution. Yuan et al. [126] evaluated PXcontaining SLNs with different lipids, namely, monostearin, stearic acid, glycerol tristearate, and ATO 888. Cellular uptake studies of NPs were performed in A549 cells, resulting in uptake in the order of glycerol tristearate $>$ monostearin $>$ stearic acid $>$ ATO 888 . Since the drug entrapment efficiency was low for glycerol tristearate SLNs, the monostearin SLNs were the optimized formulations for PX delivery. The Murthy laboratory [127,128] used either glyceryl palmitostearate or glyceryl monostearate as the lipid matrix, and poloxamer 407 as the surfactant to encapsulate PX. Arica-Yegin [129] developed PX-loaded SLNs with phospholipids and sucrose fatty acid esters using either solvent or ultrasound emulsification method.

The Mumper laboratory [130,131] developed PX-encapsulated SLNs using emulsifying wax as the oil phase, and Brij 78 as the surfactant. The drug loading achieved $150 \mu \mathrm{g} / \mathrm{mL}$ final PX concentration with entrapment efficiency about $50 \%$. The NPs showed significantly enhanced toxicity in P-gp resistant HCT-15 cells, and also increased brain uptake using an in-situ rat brain perfusion model. The in vivo antitumor efficacy of PX-loaded NPs was conducted in a 
resistant HCT-15 xenograft mouse model and NPs demonstrated slower tumor growth rate compared to Taxol. Taken together, this PXloaded NP formulation had the potential to overcome P-gp-mediated MDR both in vitro and in vivo. Later, novel PX SLNs were developed using glyceryl tridodecanoate as the core lipid. Compared to previous emulsifying wax-based SLNs, the novel PX-loaded NPs had higher drug entrapment efficiency of $85 \%$, superior physical stability, slower and sustained release profile and even elimination of initial drug burst release $[132,133]$.

\section{Lipid Nanocapsules}

Benoit and colleagues [134-136] has developed PX-loaded lipid nanocapsules, which were composed of Labrafac $^{\circledR}$ (triglycerides), Lipoïd $^{\circledR}$ S75-3 (soybean lecithin), and Solutol ${ }^{\circledR}$ HS15 (Polyethylene glycol 660 12-hydroxystearate), sodium chloride, and water. The NP formulations demonstrated longer circulation time in blood, and slower distribution and elimination in Wistar rats. Furthermore, the PX-loaded NPs could significantly increase the life span as compared to all controls. Captex ${ }^{\circledR} 8000$ (glyceryl tricaprylate) was utilized instead of Labrafac ${ }^{\circledR}$ to engineer another version of lipid nanocapsule formulation, which also showed benefits over that of Taxol in vivo and potentially overcame P-gp-mediated drug resistance [137-140].

The Mumper laboratory [132,133] developed PX-loaded nanocapsules using a warm microemulsion precursor method, which consisted of Miglyol 812 as the oil phase, and Brij 78 and TPGS as the surfactants [141]. This lipid-based nanocapsule system overcame P-gpmediated drug resistance both in vitro and in vivo, and the mechanisms were investigated which included 1) increase drug cell uptake and retention; 2) inhibit P-gp; and 3) deplete ATP. Ho et al. [142,143] prepared a complex self-assembled immuno-nanocapsule formulation. First, the PLGA polymeric core was formed to encapsulate PX with the aid of lecithin and DSPE-PEG ${ }_{2000}$. Another lipid layer with DSPC and DSPE-PEG ${ }_{2000}$ was further coated on the PLGA core to load a second drug of combretastatin A4 (a vascular disrupting agent). Moreover, RGD peptide targeting ligand was conjugated on the surface of the lipid layer. This dual-drug loaded nanocapsule delivery system was shown to be promising in cancer therapy. Bae et al. [144] designed PX-loaded PEO-PPO-PEO/PEG nanocapsules with a targeting ligand of folic acid and a lipid of Lipiodol (an iodized ethyl ester of fatty acid). In order to do so, Lipiodol, PEO-PPO-PEO co-polymer, and amine functionalized six-arm-branched PEG were used to first form Lipiodol-encapsulated PEO-PPO-PEO/PEG nanocapsules using emulsion-solvent evaporation method. PX was then loaded into the nanocapsules and the targeting ligand of folic acid was conjugated on the surface of NPs. PX loading capacity in the NPs was about 5-fold higher than without the oil phase of Lipiodol. This polymeric nanocapsule formulation with an oil core was served as a novel platform for water-insoluble drug delivery. Another novel folate-mediated solid-liquid lipid NP formulation was designed and developed to deliver PX. The idea was from that the combination of solid lipids and liquid oils could increase drug loading and entrapment efficiency [145]. The NPs demonstrated prolonged and sustained in vitro release and exhibited better antitumor efficacy compared to Taxol in S-180 tumor-bearing mice [145].

\section{PX Fatty Acid-Prodrug Lipid-Based Nanoparticles}

Although PX is a poorly water-soluble compound, its solubility in many lipids is also limited. Therefore, PX has the tendency to aggregate or crystallize from lipid-based NPs. To overcome the problem, modification of PX to a prodrug is a potential approach. Fatty acids could be used to conjugate to PX, improve the lipophilicity of PX and facilitate its retention in the core of lipid-based NPs.
Ansell et al. [146] synthesized series of PX prodrugs formulated in lipophilic NPs. Succinic acid and diglycolic acid were used as linkers. The diglycolic linkage offered a higher hydrolysis rate compared to succinate analogues. In in vitro cytotoxicity studies, succinate-linked prodrugs were 3-10-fold and 30-90-fold less potent than diglycolic linked prodrugs and free PX, respectively, in A2780 and MCF-7 cells. This was consistent with the fact that diglycolic linkage was more susceptible to hydrolysis. To evaluate the partition behavior of the prodrug NP formulations in vivo, NP plasma elimination studies were performed. The results showed that prodrugs in the NPs released first and then esterases cleaved the ester bond to form active PX form in their lipid NP formulations. Furthermore, the partitioning half-lives of diglycolate prodrugs from particles were increased as the aliphatic chain length increased, which indicated that the release rate of the prodrug from NPs could be adjusted by the length of aliphatic chains. Interestingly, succinate prodrug-containing NPs showed ineffective in vivo in an HT29 human colon carcinoma tumor xenograft mouse model, which was probably due to the low hydrolysis rate and the slowly released PX was not sufficient to achieve needed efficacy. In contrast, diglycolate prodrug-loaded NPs showed tumor growth inhibition and the antitumor activity was correlated with the partitioning half-lives of these prodrugs, where longer aliphatic chain length with longer partitioning half-life led to improved efficacy.

Lundberg et al. [147] synthesized a PX-oleate conjugate and incorporated it into a lipid emulsion formulation with the mean particle size of $50 \mathrm{~nm}$. The formulated PX-oleate was much more lipophilic than PX and had water solubility of $34 \mathrm{nM}$ and $\mathrm{K}_{\mathrm{o} / \mathrm{w}}$ of 8,074 versus PX water solubility of $12.8 \mu \mathrm{M}$ and $\mathrm{K}_{\mathrm{o} / \mathrm{w}}$ of 311 at $20^{\circ} \mathrm{C}$. This highly lipophilic PX-oleate conjugate in the NP formulation showed significantly greater $\mathrm{AUC}$, higher $\mathrm{C}_{\max }$, lower systemic clearance compared to PX formulated in Cremophor EL/ethanol in rabbits. Perkins et al. [148] attached a 16-carbon acyl chain to PX and this 2'-2-bro-mohexadecanoyl PX (BrC16-PX) conjugate was further incorporated into lipid-coated particles termed 'lipocores'. The particle size of BrC16-PX-containing lipocores was 50-100 $\mathrm{nm}$. In an in vivo study, the lipocores demonstrated antitumor activity in an ovarian carcinoma SCID mice model after intraperitoneal or intravenous administration, and were far less toxic than Taxol. Stevens et al. [149] synthesized a PX-2'-carbonyl-cholesterol (PX-Chol) conjugate to increase lipophilicity of PX and formulated it into a lipid NP formulation. The \% entrapment of PX-Chol was greater than $90 \%$ at a drug/lipid ratio of 1:20, which significantly improved drug loading efficiency compared to the previous PX-loaded lipid NPs with the loading efficiency of $70 \%$. In addition, the PX-Chol-incorporated NPs exhibited excellent colloidal stability. Ma et al. [150] synthesized a novel lipophilic paclitaxel derivative, 2'-behenoyl-paclitaxel (C22$\mathrm{PX}$ ), and then incorporated this conjugate into a lipid-based NP formulation for the treatment of metastatic breast cancer. The results of in vitro and in vivo experiments demonstrated that C22-PX NPs were much better tolerated, had significantly greater plasma and tumor AUCs, and superior anti-tumor efficacy compared to Taxol at MTD in a subcutaneous $4 \mathrm{~T} 1$ mouse model.

The most successful PX-fatty acid prodrug is docosahexaenoic acidPX (DHA-PX) conjugate, which currently is in phase III clinical trials [151]. DHA (22 carbon chain) is found in human milk and is known to be essential nutrition for brain development. DHA was linked to PX through an ester bond on its C-2' position. The DHA-PX conjugate did not have microtubule assembly activity and was non-toxic. It converted to active PX form when metabolized by esterases in the body. Therefore, 
the conjugate prolonged the exposure of PX and reduced the peak concentration, which allowed the administration of 4.4-fold higher doses of DHA-PX conjugate over that of PX alone in mice. In an M109 mouse tumor model, the tumor AUCs of PX from DHA-PX conjugate were 1.71- and 5.79-fold higher than those of PX when intravenously injected into mice at equimolar and equitoxic doses, respectively [152, 153]. It should be noted that DHA-PX is still formulated in Cremophor EL and ethanol, and this DHA-PX conjugate is beyond the scope of this review which is focused on PX nano-medicine delivery systems.

\section{Micro- and Nano-Emulsions}

TOCOSOL $^{\mathrm{TM}}$ is an emulsion formulation consisting of vitamin $\mathrm{E}$, TPGS, and poloxamer 407. In this formulation, PX was formulated in an o/w microemulsion with the particle size of $100 \mathrm{~nm}$ and high drug loading of $10 \mathrm{mg} / \mathrm{mL}$. TOCOSOL ${ }^{\mathrm{TM}}$ completely eliminated Cremophor EL and ethanol in Taxol formulation, and without any modification of PX molecular structure. In preclinical studies, TOCOSOL ${ }^{\mathrm{TM}}$ showed much better tolerance and significantly improved antitumor efficacy compared to Taxol in both B16 and HCT-15 tumor-bearing mouse models. After intravenous injection of TOCOSOL ${ }^{\mathrm{TM}}$ at the dose of $10 \mathrm{mg} / \mathrm{kg}$ to mice implanted with B16 cells, tumor AUC was 2.2-fold higher than that of Taxol, although plasma AUC was comparable. The phase I clinical studies confirmed that TOCOSOL ${ }^{\mathrm{TM}}$ was well tolerated and less toxic than Taxol $[154,155]$. However, all the phase III clinical trials of TOCOSOL ${ }^{\mathrm{TM}}$ were closed due to its comparable objective response rate to Taxol in women with metastatic breast cancer.

Kan et al. [156] optimized PX in an o/w emulsion with an oil blend (tributyrin, tricaproin, and tricaprylin), egg phosphatidylcholine (EPC), Tween 80 , and glycerol. The particle size of the NPs was $\sim 150 \mathrm{~nm}$ with a drug entrapment efficiency of $95 \%$. This emulsion formulation remarkably increased the life span of mice compared to Taxol in an intraperitoneal S-180 tumor-bearing mouse model. Nornoo and Chow $[157,158]$ developed two microemulsion systems of LBMW (le cithin:butanol:myvacet:water) and CMW (capmul:myvacet:water) as the carriers for PX delivery. Compared to Taxol, the release of PX was extended by $25 \%$ and 50\% in LBMW and CMW formulations, respectively. In in vivo pharmacokinetic studies in Sprague-Dawley rats, LBMW and CMW prolonged by 5 - and 2 -fold the circulation time of PX in blood, and 8- and 3-fold higher plasma AUC, respectively, over that of Taxol. The Amiji group [159] from Northeastern University formulated PX in an oral o/w nanoemulsion with pine nut oil as the oil phase, egg lecithin as the primary emulsifier, and stearylamine and deoxycholic acid to modify positive and negative charges, respectively. After oral administration, this nanoemulsion demonstrated enhanced PX concentration in the systemic circulation and greater bioavailability compared to the control aqueous solution. Later on, the same research group [160] developed a flaxseed oil containing nanoemulsion formulation, where PX and curcumin were co-encapsulated into the

\begin{tabular}{|c|c|c|c|}
\hline Polymer & Linker / Spacer & Status & Ref. \\
\hline \multirow{2}{*}{ PAMAM } & Ester / succinic acid & in-vitro & {$[161]$} \\
\cline { 2 - 4 } & Ester / FITC-folic acid & in-vitro & {$[162]$} \\
\hline \multirow{2}{*}{ Heparin } & Ester / valine, leucine, phenylalanine & in-vivo & {$[163]$} \\
\cline { 2 - 4 } & Ester / folic acid & in-vivo & {$[165]$} \\
\hline \multirow{2}{*}{ PGG } & ester & in-vivo & {$[166-168]$} \\
\cline { 2 - 4 } & Ester / PEG-RGD & in-vitro & {$[169]$} \\
\hline HA & Ester / valine, leucine, phenylalanine & in-vitro & {$[164]$} \\
\hline \multirow{2}{*}{ HPMA } & Ester / Gly-Phe-Leu-Gly & phase I/ & {$[170]$} \\
\hline PG & ester & phase III & {$[171,172]$} \\
\hline
\end{tabular}

Table 3: Summary of PX-polymer Conjugates. nanoemulsions. The addition of curcumin was to inhibit NFkB and down-regulate $\mathrm{ABC}$ transporters, and the formulation was shown to be effective in wild-type SKOV3 cells and multi-drug resistant SKOV $3_{\text {TR }}$ cells as well.

\section{Drug-Polymer Conjugates}

A summary of PX-Polymer conjugates is shown in Table 3. Poly(amidoamine) (PAMAM) dendrimer-based PX conjugates were designed and synthesized. Khandare et al. [161] prepared PAMAM dendrimer-succinic acid-PX conjugate and showed that the cytotoxicity of the conjugate was 10 -fold higher than that of free unconjugated drug in A2780 human ovarian carcinoma cells. Multifunctional PAMAM dendrimer was also engineered. The functional molecules of fluorescein isothiocyanate (FITC, an imaging agent), folic acid, and PX were conjugated to PAMAM dendrimer. This conjugate was evaluated and confirmed to have both targeted chemotherapeutic and imaging functions to cancer cells in vitro [162].

Wang et al. [163] synthesized a series heparin-PX conjugates with various single amino acid spacers, namely, valine, leucine, and phenylalanine. All the three conjugates exhibited greater cytotoxicity than that of free PX in MCF-7 cells. The hydrolysis properties of the conjugates in physiological and plasma conditions were different and the fastest hydrolysis was observed using leucine as the spacer. This heparin-PX conjugate with leucine spacer had similar antitumor efficacy compared to Taxol at a dose of $30 \mathrm{mg}$ PX/kg in an SKOV3 mouse model. Similarly, hyaluronic acid was utilized as the carrier to conjugate with PX. Different amino acids as the spacers were applied, such as valine, leucine, and phenylalanine. The conjugates exhibited increased cytotoxicity compared to free PX in MCF-7 cells [164]. A ternary conjugate of heparin-folic acid-PX was synthesized for further targeting purpose. This conjugate proved to selectively recognize folate receptor positive KB-3-1 cells and remarkably enhanced tumor inhibitory activity in a subcutaneous KB-3-1 xenograft model [165]. The Yu group [166-168] developed a novel poly(L- $\gamma$-glutamyl-glutamine $)$ PX (PGG-PX) conjugate. Interestingly, this conjugate self-assembled into NPs with the particle size of $12-15 \mathrm{~nm}$. The formulation had low toxicity in mice and its in vivo antitumor activity was superior to Abraxane ${ }^{\circledR}$ in multiple tumor models. Later, a more complex targeted PGG-PX-PEG-RGD conjugate was synthesized to further improve the therapeutic index [169].

An HPMA-co-polymer-PX conjugate (PNU 166945) was developed by covalent bonding of $\mathrm{PX}$ at its 2'-OH position with an enzymatic degradable tetrapeptide linker of Gly-Phe-Leu-Gly. The polymer/PX ratio was about $19 / 1(\mathrm{w} / \mathrm{w})$. It was the first polymer-PX conjugate that entered phase I clinical trials. In the phase I studies, PNU 166945 was administered by $1 \mathrm{~h}$ infusion every 3 weeks to a small patient cohort of 12 patients. One patient had a partial response with advanced breast cancer. However, PNU 166945 demonstrated neurotoxicity and because of that the clinical studies were discontinued [170].

The most promising polymer-PX conjugate to date is undoubtedly the poly (L-glutamic acid)-PX conjugate (PG-PX, CT-2103), where $\mathrm{PG}$ is conjugated to 2'-OH position of $\mathrm{PX}$ via an ester linkage. The conjugate showed significantly better antitumor efficacy compared to Taxol in several tumor-bearing mouse models, and in some cases it completely eliminated tumors. In addition, the MTD and tumor uptake of CT-2013 were about 2- and 5-fold higher than Taxol, respectively, and prolonged circulation time was also observed in mouse tumor models. Currently, CT-2103 is in phase III clinical trials [171,172]. 


\section{Inorganic Nanoparticles}

\section{Gold Nanoparticles}

Gibson et al. [173] covalently attached PX with a hexaethylene glycol linker to phenol-terminated gold NPs. Thermogravimetric analysis indicated there was about 70 PX molecules per 1 gold NP. The NPs were characterized by various traditional analytical techniques, such as NMR, TEM, SEC, etc., and concluded that the PX-coated gold NPs were able to be engineered in a controlled manner and this offered an alternative platform for PX delivery. Oh et al. [174] described a novel PX-loaded gold/chitosan/pluronic NP system. To prepare the modified gold NPs, PX was first dissolved in Tween 80 and this PX/ Tween 80 was then dispersed into placebo gold NPs. Next, chitosan was introduced to the mixture to form PX-loaded gold/chitosan NPs via ionic interaction. The gold/chitosan NPs were then freeze-dried with pluronic F127 to obtain powders. The results showed that PX was released up to $90 \%$ from NPs over a 12 -day period with a minimum initial burst release. Therefore, PX-loaded gold NPs may serve as an effective nanomedicine for better cancer therapy. Heo and co-workers [175] described a complex PX-functionalized gold NP system with PEG, biotin, and rhodamine B-linked $\beta$-CD. In the system, PEG served as an anti-fouling shell, biotin as a targeting ligand, rhodamine $\mathrm{B}$ as a fluorescent marker, $\beta-\mathrm{CD}$ as the carrier for both $\mathrm{PX}$ and rhodamine B. The PX-loaded gold NP demonstrated more potent cytotoxicity in cancer cell lines of HeLa, A549, and MG63 than the normal NIH3T3 cells, which indicated its potential use in cancer treatment.

\section{Magnetic Nanoparticles (MNPs)}

Hua et al. [176] designed poly(aniline-co-sodium N-(1-one-butyric acid) aniline) (SPAnNa) coated $\mathrm{Fe}_{3} \mathrm{O}_{4}$ MNPs. PX was immobilized onto the surface of MNPs to form SPAnNa/MNPs-bound-PX (bound-PX). The drug loading achieved up to $302.75 \mu \mathrm{g}$ per mg of SPAnNa/MNPs. The bound-PX demonstrated higher stability than free PX at $37^{\circ} \mathrm{C}$ (halflife of 57 vs. $19 \mathrm{~h}$ ). Importantly, this bound-PX exhibited more potent toxicity compared to free PX in both PC3 and CWR22R prostate cancer cells. Moreover, the cell inhibition activity was even enhanced when a magnetic field was applied. All together, these results indicated that this magnetic delivery system had the potential to treat prostate cancer. Hwu and co-workers [177] developed PX-containing Fe-MNPs. The aqueous solubility of PX in PX-Fe-MNPs increased 780-fold (312 vs $0.4 \mu \mathrm{g} / \mathrm{mL}$ ). The beauty of the design was that phosphodiester moiety was incorporated as the linker of PX. Since dephosphorylation took place easier in tumor cells, PX was able to be released from NPs once the NPs reached the tumor site. This was confirmed by the fact that $91 \%$ free PX was hydrolyzed and released from NPs after 10 days. In vitro cytotoxicity studies were conducted in both human cancer OECM1 cells and human normal HUVEC cells. It was found that PX-Fe-MNPs were 14,050-fold more toxic in cancer cells than human normal cells ( IC $_{50}$ of $5.03 \times 10^{-7}$ vs $3.58 \times 10^{-3} \mu \mathrm{g} / \mathrm{mL}$ ).

\section{Others}

\section{Carbon Nanotubes (CNTs)}

Liu et al. [178] conjugated PX to branched PEG on single-walled carbon nanotubes to form water-soluble CNTs. This PX-loaded PEGfunctionalized CNT exhibited excellent stability and PX was released from nanotubes by esterases present in the body. The formulation demonstrated significantly improved antitumor activity compared to Taxol at the dose of $5 \mathrm{mg} / \mathrm{kg}$ in a $4 \mathrm{~T} 1$ breast cancer mouse model, which was supported by the prolonged circulation time in blood and 10-fold enhanced tumor uptake. Similarly, Lay and co-workers [179] loaded PX on the surface of PEG-functionalized single- or multiplewalled CNTs. The drug loading was $26 \%$ and $36 \%(w / w)$ for singleand multiple-walled CNTs, respectively. In-vitro cytotoxicity studies in HeLa and MCF-7 cells showed both PX-loaded CNTs significantly increased cell death compared to free PX while the placebo CNTs were non-toxic. Recently Sobhani et al. [180] functionalized multiple-walled CNTs using hyperbranched poly citric acid and conjugated PX on the CNTs. The drug loading was about $38 \%(\mathrm{w} / \mathrm{w})$. This formulation was more toxic than Taxol in both A549 and SKOV3 cells while the blank CNTs had a negligible effect.

\section{Nanocrystals}

The Huang group [181] developed a PX nanocrystal formulation using TPGS as the sole excipient. The utilization of TPGS in the formulation was to stabilize PX nanocrystals as well as reverse MDR. The average particle size of nanocrystals was $40 \times 150 \mathrm{~nm}$. The nanocrystals exhibited slower and sustained release than Taxol, and enhanced in vitro cytotoxicity and in vivo antitumor efficacy in resistant NCI/ADR-RES tumor models. PX/Pluronic F127 nanocrystals were also engineered and reported. The drug loading and entrapment efficiency were high and this formulation demonstrated significantly tumor growth inhibition activity in both human lung cancer and murine breast cancer mouse models [182]. Interestingly, it was found that the micellization of pluronic F127 enhanced at elevated temperature, which was because the critical micelle concentration (CMC) of pluronic F127 decreased at higher temperature. Therefore, the increased temperature during preparation procedure may provide better stability of nanocrystals [183].

\section{Cyclodextrin (CD) Nanoparticles}

Zhang et al. [184] synthesized a CD-functionalized hyperbranched polyglycerol (HPG) as the carrier for PX delivery. The HPG-g-CD self-assembled micelles in water with the particle size of 200-300 nm. PX-loaded HPG-g-CD had high drug loading $(\sim 8 \%$, w/w) and entrapment efficiency (82-88\%), and slow and sustained release profile. A $\beta$-CD derivative, heptakis (6-O-hexanoyl) cyclomaltoheptaose- $\beta$ CD (6-O-CAPRO- $\beta$-CD) was synthesized to stabilize PX NPs with high drug loading and reduce the burst release. Since the carrier of 6-O-CAPRO- $\beta$-CD demonstrated low hemolysis and cytotoxicity, it may serve as an alternative formulation for current Taxol [185-187].

\section{Nanogel}

Lee et al. [188] developed PX-loaded injectable in situ-forming gel with mPEG-PCL diblock co-polymer. The formulation was in a liquid state at room temperature but rapidly formed gel at body temperature. PX in the gel was released in a sustained manner for more than 2 weeks in vitro. This PX injectable depot had significantly improved antitumor efficacy compared to Taxol, saline (control), and placebo gel in a B16F10 tumor-bearing mouse model upon intratumoral injection. OncoGel is a PX injectable depot developed by MacroMed Inc (Sandy, Utah) for local tumor treatment. The gel was composed of the thermosensitive triblock co-polymer of PLGA-PEG-PLGA, which transformed to a water-insoluble hydrogel at body temperature. It had sustained release properties for up to 6 weeks upon injection. OncoGel was proven to be safe and reduced systemic exposure of PX because PX concentration in plasma was negligible after single injection into solid tumor site. Currently, it is in phase II clinical trials $[189,190]$.

\section{ANG 1005}

ANG 1005 is a PX-peptide conjugate, which consists of three PX 


\begin{tabular}{|c|c|c|c|c|}
\hline Name & Delivery Strategy & Company & Clinical Stage & Disease Condition Used / Treated \\
\hline Abraxane & protein-bound particle & Abraxis Bioscience (now Celgene) & approved & metastatic breast cancer / NSCLC \\
\hline NK 105 & polymeric micelle & NanoCarrier & phase III & metastatic breast cancer / stomach cancer \\
\hline CT-2103 & polymeric conjugate & Cell Therapeutics & phase III & ovarian cancer \\
\hline Genexol-PM & polymeric micelle & Samyang & phase III & metastatic breast cancer \\
\hline Tocosol & emulsion & Sonus Pharmaceuticals & phase III / discontinued & metastatic breast cancer \\
\hline ANG 1005 & peptide-bound conjugate & Angiochem & phase II & glioma \\
\hline EndoTag & cationic liposome & MediGene & phase II & metastatic breast cancer / pancreatic cancer \\
\hline Paxceed & polymeric micelle & Angiotech Pharmaceuticals & phase II & psoriasis \\
\hline LEP-ETU & liposome & NeoPharm & phase II & metastatic breast cancer \\
\hline OncoGel & gel & Protherics & phase II & esophageal cancer / primary brain cancer \\
\hline PNU166945 & polymeric conjugate & Pfizer & phase I/ discontinued & - \\
\hline
\end{tabular}

Table 4: Summary of PX-loaded NPs in Clinical Development.

molecules coupled to one molecule of a novel peptide, Angiopep-2. Angiopep-2 is a 19 amino-acid peptide which targets low-density lipoprotein receptor-related protein 1 (LRP1) receptor to facilitate PX to across BBB for the treatment of brain disease. In preclinical studies, ANG 1005 demonstrated better antitumor efficacy and increased survival time in several tumor-bearing mouse models. Currently, it is in phase II clinical trials $[191,192]$.

\section{Conclusions and Future Perspective}

$\mathrm{PX}$ is one of the most effective anticancer drugs ever developed. It is active against a broad range of cancers. However, the current Taxol formulations have issues related to the use of Cremophor EL and ethanol. Since nano-delivery systems could have the potential to be free of Cremophor EL and ethanol, enhance PX solubility, improve PX pharmacokinetic profiles in vivo, decrease its side effects, passively or actively target to tumor sites due to the EPR effect and the use of targeting ligands, respectively, nanotechnology is a very active research area in both academic and industrial settings. Therefore, various types of PX nano-delivery systems have been developed as discussed in this review, such as polymeric nanoparticles, lipid-based formulations, polymer conjugates, inorganic nanoparticles, carbon nanotubes, nanocrystals, and cyclodextrin nanoparticles. To date, the PX albumin-bound NPs (Abraxane ${ }^{\mathbb{R}}$ ) have been approved by the FDA for the treatment of metastatic breast cancer and NSCLC, and there are a number of novel PX NP formulations in clinical trials (Table 4). Some of them have demonstrated certain advantages in terms of toxicity, such as lower incidence of hypersensitivity reactions, myelosuppression, etc. However, whether these novel formulations may improve survival is largely unknown.

Although it has been established that nano-delivery systems are feasible and promising in cancer therapy, there are a lot challenges as well to hinder the application of these systems. First, it is difficult to fully characterize the physicochemical properties of NP systems and it seems that every NP delivery system is unique; and related to this, the large-scale manufacturing of NP will be another challenge. Second, the lack understanding of NP stability and drug release mechanisms from NP formulations in-vivo, or in general, the fate of the drug as well as the vehicles in human body. Third, the theory of EPR effect is debatable and now it is generally considered that tumor leakage is cancer-type dependent, and nano-delivery systems with particle sizes below $40 \mathrm{~nm}$ are desirable to have a better chance to passively target tumors. Fourth, it is important to search for new tumor targeting ligands which can be precisely characterized and exclusively expressed in the targeted organs. Fifth, the long-term toxicity of these nano-materials in the human body must be investigated. In regard to this mandate, biodegradable and non-toxic polymers or lipids are less toxic and likely have a better opportunity. In addition, the price of NP delivery systems is expected to be high. Nevertheless, the future of NP delivery systems remains promising and is sure to have a role in improving cancer therapy.

\section{Acknowledgements}

The authors are supported, in part, by Award Number U54 CA151652 from the National Cancer Institute. The content is solely the responsibility of the authors and does not necessarily represent the official views of the National Cancer Institute or the National Institutes of Health.

\section{References}

1. Jordan MA, Wilson $L$ (2004) Microtubules as a target for anticancer drugs. Nat Rev Cancer 4: 253-265.

2. Gelderblom H, Verweij J, Nooter K, Sparreboom A (2001) Cremophor EL: the drawbacks and advantages of vehicle selection for drug formulation. Eur $J$ Cancer 37: 1590-1598.

3. Sparreboom A, van Tellingen O, Nooijen WJ, Beijnen JH (1996) Nonlinea pharmacokinetics of paclitaxel in mice results from the pharmaceutical vehicle Cremophor EL. Cancer Res 56: 2112-2115.

4. Gallo JM, Li S, Guo P, Reed K, Ma J. (2003) The effect of P-glycoprotein on paclitaxel brain and brain tumor distribution in mice. Cancer Res. 63: 51145117

5. Berg SL, Tolcher A, O'Shaughnessy JA, Denicoff AM, Noone M, et al. (1995) Effect of R-verapamil on the pharmacokinetics of paclitaxel in women with breast cancer. J Clin Oncol 13: 2039-2042.

6. Fracasso PM, Westervelt P, Fears CL, Rosen DM, Zuhowski EG, et al. (2000) Phase I study of paclitaxel in combination with a multidrug resistance modulator PSC 833 (Valspodar), in refractory malignancies. J Clin Oncol 18: 1124-1134.

7. Storm G, Belliot SO, Daemen T, Lasic DD (1995) Surface modification of nanoparticles to oppose uptake by the mononuclear phagocyte system. Advanced Drug Delivery Reviews 17: 31-48.

8. Alexis F, Pridgen EM, Langer R, Farokhzad OC (2010) Nanoparticle technologies for cancer therapy. Handb Exp Pharmacol : 55-86.

9. Wang J, Sui M, Fan W (2010) Nanoparticles for tumor targeted therapies and their pharmacokinetics. Curr Drug Metab 11: 129-141.

10. Haley B, Frenkel E (2008) Nanoparticles for drug delivery in cancer treatment Urol Oncol 26: 57-64.

11. Suri SS, Fenniri H, Singh B. (2007) Nanotechnology-based drug delivery systems. J Occup Med Toxicol 2: 16.

12. Jain RK, Stylianopoulos T (2010) Delivering nanomedicine to solid tumors. Nat Rev Clin Oncol 7: 653-664.

13. Jin C, Wu H, Liu J, Bai L, Guo G (2007) The effect of paclitaxel-loaded nanoparticles with radiation on hypoxic MCF-7 cells. J Clin Pharm Ther 32 $41-47$

14. Jin C, Bai L, Wu H, Liu J, Guo G, et al. (2008) Paclitaxel-loaded poly(D,L lactide-co-glycolide) nanoparticles for radiotherapy in hypoxic human tumo cells in vitro. Cancer Biol Ther 7: 911-916.

15. Danhier F, Lecouturier N, Vroman B, Jérôme C, Marchand-Brynaert J, et al. 
(2009) Paclitaxel-loaded PEGylated PLGA-based nanoparticles: in vitro and in vivo evaluation. J Control Release 133: 11-17

16. Fonseca C, Simões S, Gaspar R (2002) Paclitaxel-loaded PLGA nanoparticles: preparation, physicochemical characterization and in vitro anti-tumoral activity. J Control Release 83: 273-286.

17. Dong Y, Feng SS (2007) Poly(D,L-lactide-co-glycolide) (PLGA) nanoparticles prepared by high pressure homogenization for paclitaxel chemotherapy. Int J Pharm 342: 208-214.

18. Jin C, Bai L, Wu H, Song W, Guo G, et al. (2009) Cytotoxicity of paclitaxe incorporated in PLGA nanoparticles on hypoxic human tumor cells. Pharm Res 26: $1776-1784$

19. Kim BS, Kim CS, Lee KM (2008) The intracellular uptake ability of chitosancoated poly(D,L-lactideco-glycolide) nanoparticles. Arch Pharm Res 31: 10501054.

20. Chakravarthi SS, Robinson DH (2011) Enhanced cellular association of paclitaxel delivered in chitosan-PLGA particles. Int J Pharm 409: 111-120.

21. Bhardwaj V, Ankola D, Gupta S, Schneider M, Lehr CM, et al. (2009) PLGA nanoparticles stabilized with cationic surfactant: safety studies and application in oral delivery of paclitaxel to treat chemical-induced breast cancer in rat. Pharm Res 26: 2495-2503.

22. Mei L, Sun H, Jin X, Zhu D, Sun R, et al. (2007) Modified paclitaxel-loaded nanoparticles for inhibition of hyperplasia in a rabbit arterial balloon injury model. Pharm Res 24: 955-962.

23. Vandervoort J, Ludwig A (2002) Biocompatible stabilizers in the preparation of PLGA nanoparticles: a factorial design study. Int J Pharm 238: 77-92.

24. Mu L, Feng SS (2003) A novel controlled release formulation for the anticance drug paclitaxel (Taxol): PLGA nanoparticles containing vitamin E TPGS. J Control Release 86: 33-48.

25. Feng SS, Mu L, Win KY, Huang G (2004) Nanoparticles of biodegradable polymers for clinical administration of paclitaxel. Curr Med Chem 11: 413-424.

26. Feng SS, Zeng W, Teng Lim Y, Zhao L, Yin Win K, et al. (2007) Vitamin E TPGS-emulsified poly(lactic-co-glycolic acid) nanoparticles for cardiovascular restenosis treatment. Nanomedicine (Lond) 2: 333-344.

27. Zhao L, Feng SS (2010) Enhanced oral bioavailability of paclitaxel formulated in vitamin E-TPGS emulsified nanoparticles of biodegradable polymers: in vitro and in vivo studies. J Pharm Sci 99: 3552-3560.

28. Liu Y, Pan J, Feng SS (2010) Nanoparticles of lipid monolayer shell and biodegradable polymer core for controlled release of paclitaxel: effects of surfactants on particles size, characteristics and in vitro performance. Int $J$ Pharm 395: 243-250

29. Feng SS, Huang G (2001) Effects of emulsifiers on the controlled release of paclitaxel (Taxol) from nanospheres of biodegradable polymers. J Control Release 71: 53-69.

30. Dong Y, Feng SS (2005) Poly(D,L-lactide-co-glycolide)/montmorillonite nanoparticles for oral delivery of anticancer drugs. Biomaterials 26: 6068-6076.

31. Sun B, Ranganathan B, Feng SS (2008) Multifunctional poly(D,L-lactideco-glycolide)/montmorillonite (PLGA/MMT) nanoparticles decorated by Trastuzumab for targeted chemotherapy of breast cancer. Biomaterials 29: 475-486.

32. Wang Z, Chui WK, Ho PC (2011) Nanoparticulate delivery system targeted to tumor neovasculature for combined anticancer and antiangiogenesis therapy. Pharm Res 28: 585-596.

33. Shah N, Chaudhari K, Dantuluri P, Murthy RS, Das S (2009) Paclitaxel-loaded PLGA nanoparticles surface modified with transferrin and Pluronic ${ }^{\circledR}$ P85, an in vitro cell line and in vivo biodistribution studies on rat model. J Drug Target 17: $533-542$

34. Sahoo SK, Ma W, Labhasetwar V (2004) Efficacy of transferrin-conjugated paclitaxel-loaded nanoparticles in a murine model of prostate cancer. Int $J$ Cancer 112: 335-340.

35. Sahoo SK, Labhasetwar V (2005) Enhanced antiproliferative activity of transferrin-conjugated paclitaxel-loaded nanoparticles is mediated via sustained intracellular drug retention. Mol Pharm 2: 373-383.

36. Dong Y, Feng SS (2004) Methoxy poly(ethylene glycol)-poly(lactide) (MPEGPLA) nanoparticles for controlled delivery of anticancer drugs. Biomaterials 25 2843-2849.
37. Dong Y, Feng SS (2007) In vitro and in vivo evaluation of methoxy polyethylene glycol-polylactide (MPEG-PLA) nanoparticles for small-molecule drug chemotherapy. Biomaterials 28: 4154-4160.

38. Dong Y, Feng SS (2006) Nanoparticles of poly(D,L-lactide)/methoxy poly(ethylene glycol)-poly(D,L-lactide) blends for controlled release of paclitaxel. J Biomed Mater Res A 78: 12-19.

39. Zhang Z, Feng SS (2006) In vitro investigation on poly(lactide) Tween 80 copolymer nanoparticles fabricated by dialysis method for chemotherapy. Biomacromolecules 7: 1139-1146.

40. Zhang Z, Feng SS (2006) Nanoparticles of poly(lactide)/vitamin E TPGS copolymer for cancer chemotherapy: synthesis, formulation, characterization and in vitro drug release. Biomaterials 27: 262-270.

41. Zhang Z, Feng SS (2006) Self-assembled nanoparticles of poly(lactide) --vitamin E TPGS copolymers for oral chemotherapy. Int J Pharm 324: 191 198

42. Zhang Z, Lee SH, Gan CW, Feng SS (2008) In vitro and in vivo investigation on PLA-TPGS nanoparticles for controlled and sustained small molecule chemotherapy. Pharm Res 25: 1925-1935

43. Zhang Z, Feng SS (2006) The drug encapsulation efficiency, in vitro drug release, cellular uptake and cytotoxicity of paclitaxel-loaded poly(lactide)tocopheryl polyethylene glycol succinate nanoparticles. Biomaterials 27: 40254033.

44. Pan J, Feng SS (2008) Targeted delivery of paclitaxel using folate-decorated poly(lactide)-vitamin E TPGS nanoparticles. Biomaterials 29: 2663-2672.

45. Zhang L, Hu CH, Cheng SX, Zhuo RX (2010) Hyperbranched amphiphilic polymer with folate mediated targeting property. Colloids and Surfaces B: Biointerfaces 79: 427-433.

46. Cirstoiu-Hapca A, Buchegger F, Bossy L, Kosinski M, Gurny R, et al. (2009) Nanomedicines for active targeting: physico-chemical characterization of paclitaxel-loaded anti-HER2 immunonanoparticles and in vitro functional studies on target cells. Eur J Pharm Sci 38: 230-237.

47. Patil YB, Toti US, Khdair A, Ma L, Panyam J (2009) Single-step surface functionalization of polymeric nanoparticles for targeted drug delivery Biomaterials 30: 859-866.

48. Jie P, Venkatraman SS, Min F, Freddy BY, Huat GL (2005) Micelle-like nanoparticles of star-branched PEO-PLA copolymers as chemotherapeutic carrier. J Control Release 110: 20-33.

49. Xiao L, Xiong X, Sun X, Zhu Y, Yang H, et al. (2011) Role of cellular uptake in the reversal of multidrug resistance by PEG-b-PLA polymeric micelles. Biomaterials 32: 5148-5157.

50. Gaucher G, Poreba M, Ravenelle F, Leroux JC (2007) Poly(N-vinylpyrrolidone)-block-poly(D,L-lactide) as polymeric emulsifier for the preparation of biodegradable nanoparticles. J Pharm Sci 96: 1763-1775.

51. Liang HF, Chen CT, Chen SC, Kulkarni AR, Chiu YL, et al. (2006) Paclitaxelloaded poly(gamma-glutamic acid)-poly(lactide) nanoparticles as a targeted drug delivery system for the treatment of liver cancer. Biomaterials 27: 2051 2059.

52. He G, Ma LL, Pan J, Venkatraman S (2007) ABA and BAB type triblock copolymers of PEG and PLA: a comparative study of drug release properties and "stealth" particle characteristics. Int J Pharm 334: 48-55.

53. Venkatraman SS, Jie P, Min F, Freddy BY, Leong-Huat G (2005) Micellelike nanoparticles of PLA-PEG-PLA triblock copolymer as chemotherapeutic carrier. Int J Pharm 298: 219-232.

54. Zhang X, Jackson JK, Burt HM (1996) Development of amphiphilic diblock copolymers as micellar carriers of Taxol. Int J Pharm 132: 195-206.

55. Ehrlich A, Booher S, Becerra Y, Borris DL, Figg WD, et al. (2004) Micella paclitaxel improves severe psoriasis in a prospective phase II pilot study. J Am Acad Dermatol 50: 533-540.

56. Kim TY, Kim DW, Chung JY, Shin SG, Kim SC, et al. (2004) Phase I and pharmacokinetic study of Genexol-PM, a cremophor-free, polymeric micelleformulated paclitaxel, in patients with advanced malignancies. Clin Cancer Res 10: $3708-3716$

57. Kim SC, Kim DW, Shim YH, Bang JS, Oh HS, et al. (2001) In vivo evaluation of polymeric micellar paclitaxel formulation: toxicity and efficacy. J Control Release 72: 191-202. 
58. Lim WT, Tan EH, Toh CK, Hee SW, Leong SS, et al. (2010) Phase pharmacokinetic study of a weekly liposomal paclitaxel formulation (GenexolPM) in patients with solid tumors. Ann Oncol 21: 382-388.

59. Lee KS, Chung HC, Im SA, Park YH, Kim CS, et al. (2008) Multicenter phase II trial of Genexol-PM, a Cremophor-free, polymeric micelle formulation of paclitaxel, in patients with metastatic breast cancer. Breast Cancer Res Treat 108: 241-250.

60. Saif MW, Podoltsev NA, Rubin MS, Figueroa JA, Lee MY, et al. (2010) Phase II clinical trial of paclitaxel loaded polymeric micelle in patients with advanced pancreatic cancer. Cancer Invest 28: 186-194

61. Deshpande D, Devalapally H, Amiji MM (2008) Enhancement in anti-proliferative effects of paclitaxel in aortic smooth muscle cells upon co-administration with ceramide using biodegradable polymeric nanoparticles. Pharm Res 25: 1936 1947.

62. van Vlerken LE, Duan Z, Seiden MV, Amiji MM (2007) Modulation of intracellular ceramide using polymeric nanoparticles to overcome multidrug resistance in cancer. Cancer Res 67: 4843-4850.

63. Devalapally H, Duan Z, Seiden MV, Amiji MM. (2007) Paclitaxel and ceramide co-administration in biodegradable polymeric nanoparticulate delivery system to overcome drug resistance in ovarian cancer. Int J Cancer 121: 1830-1838.

64. Devalapally H, Duan ZF, Seiden MV, Amiji MM. (2008) Modulation of drug resistance in ovarian adenocarcinoma by enhancing intracellular ceramide using tamoxifen-loaded biodegradable polymeric nanoparticles. Clin Cance Res 14: 3193-3203.

65. Yadav S, van Vlerken E, Little S, Amiji MM (2009) Evaluations of combination MDR-1 gene silencing and paclitaxel administration in biodegradable polymeric nanoparticle formulations to overcome multidrug resistance in cancer cells. Cancer Chemother Pharmacol 63: 711-722.

66. Forrest M, Yáñez J, Remsberg C, Ohgami Y, Kwon G, et al. (2008) Paclitaxe prodrugs with sustained release and high solubility in poly(ethylene glycol)b-poly ( $\varepsilon$-caprolactone) micelle nanocarriers: pharmacokinetic disposition, tolerability, and cytotoxicity. Pharm Res 25: 194-206.

67. Xin H, Chen L, Gu J, Ren X, Wei Z, et al. (2010) Enhanced anti-glioblastoma efficacy by PTX-loaded PEGylated poly( $\varepsilon$-caprolactone) nanoparticles: in vitro and in vivo evaluation. Int J Pharm 402: 238-247.

68. Xin H, Jiang X, Gu J, Sha X, Chen L, et al. (2011) Angiopep-conjugated poly(ethylene glycol)-co-poly( $\varepsilon$-caprolactone) nanoparticles as dual-targeting drug delivery system for brain glioma. Biomaterials 32: 4293-4305.

69. Wang C, Wang Y, Wang Y, Fan M, Luo F, et al. (2011) Characterization pharmacokinetics and disposition of novel nanoscale preparations of paclitaxel. Int J Pharm 414: 251-259.

70. Chen S, Zhang XZ, Cheng SX, Zhuo RX, Gu ZW (2008) Functionalized amphiphilic hyperbranched polymers for targeted drug delivery. Biomacromolecules 9: 2578-2585.

71. Zhang Y, Tang L, Sun L, Bao J, Song C, et al. (2010) A novel paclitaxel-loaded poly(epsilon-caprolactone)poloxamer 188 blend nanoparticle overcoming multidrug resistance for cancer treatment. Acta Biomater 6: 2045-2052.

72. Ma GL, Yang J, Zhang LH, Song CX. (2010) Effective antitumor activity of paclitaxel-loaded poly (epsilon-caprolactone)/pluronic F68 nanoparticles after intratumoral delivery into the murine breast cancer model. Anticancer Drugs 21: $261-269$

73. Zhu Z, Li Y, Li X, Li R, Jia Z, et al. (2010) Paclitaxel-loaded poly(Nvinylpyrrolidone)-b-poly(epsilon-caprolactone) nanoparticles: preparation and antitumor activity in vivo. J Control Release 142: 438-446.

74. Sheikh F, Barakat N, Kanjwal M, Aryal S, Khil M, et al. (2009) Novel selfassembled amphiphilic poly( $\varepsilon$-caprolactone)-grafted-poly(vinyl alcohol) nanoparticles: hydrophobic and hydrophilic drugs carrier nanoparticles. Journal of Materials Science: Materials in Medicine 20: 821-831.

75. Lee SC, Kim C, Kwon IC, Chung H, Jeong SY (2003) Polymeric micelles of poly(2-ethyl-2-oxazoline)-block-poly( $\varepsilon$-caprolactone) copolymer as a carrier for paclitaxel. J Control Release 89: 437-446.

76. Wang YC, Liu XQ, Sun TM, Xiong MH, Wang J (2008) Functionalized micelles from block copolymer of polyphosphoester and poly(epsilon-caprolactone) for receptor-mediated drug delivery. J Control Release 128: 32-40.

77. Sun TM, Du JZ, Yao YD, Mao CQ, Dou S, et al. (2011) Simultaneous delivery of
siRNA and paclitaxel via a "two-in-one" micelleplex promotes synergistic tumor suppression. ACS Nano 5: 1483-1494.

78. Kim K, Kim JH, Park H, Kim YS, Park K, et al. (2010) Tumor-homing multifunctional nanoparticles for cancer theragnosis: simultaneous diagnosis, drug delivery, and therapeutic monitoring. J Control Release 146: 219-227.

79. Saravanakumar G, Min KH, Min DS, Kim AY, Lee CM, et al. (2009) Hydrotropic oligomer-conjugated glycol chitosan as a carrier of paclitaxel: synthesis characterization, and in vivo biodistribution. J Control Release 140: 210-217.

80. Trickler WJ, Nagvekar AA, Dash AK (2008) A novel nanoparticle formulation for sustained paclitaxel delivery. AAPS PharmSciTech 9: 486-493.

81. Jang MK, Jeong YI, Nah JW (2010) Characterization and preparation of coreshell type nanoparticle for encapsulation of anticancer drug. Colloids Surf B Biointerfaces 81: 530-536.

82. Park JS, Han TH, Lee KY, Han SS, Hwang JJ, et al. (2006) N-acetyl histidineconjugated glycol chitosan self-assembled nanoparticles for intracytoplasmic delivery of drugs: endocytosis, exocytosis and drug release. J Control Release 115: $37-45$.

83. Hu FQ, Ren GF, Yuan H, Du YZ, Zeng S (2006) Shell cross-linked stearic acid grafted chitosan oligosaccharide self-aggregated micelles for controlled release of paclitaxel. Colloids Surf B Biointerfaces 50: 97-103.

84. Paál K, Müller J, Hegedûs L (2001) High affinity binding of paclitaxel to human serum albumin. Eur J Biochem 268: 2187-2191.

85. Vogel SM, Minshall RD, Pilipovic M, Tiruppathi C, Malik AB (2001) Albumin uptake and transcytosis in endothelial cells in vivo induced by albumin-binding protein. Am J Physiol Lung Cell Mol Physiol 281: L1512-L1522.

86. Green MR, Manikhas GM, Orlov S, Afanasyev B, Makhson AM, et al. (2006) Abraxane, a novel Cremophor-free, albumin-bound particle form of paclitaxe for the treatment of advanced non-small-cell lung cancer. Ann Oncol 17: 1263 1268

87. Petrelli F, Borgonovo K, Barni S (2010) Targeted delivery for breast cance therapy: the history of nanoparticle-albumin-bound paclitaxel. Expert Opin Pharmacother 11: 1413-1432.

88. Hawkins MJ, Soon-Shiong P, Desai N (2008) Protein nanoparticles as drug carriers in clinical medicine. Adv Drug Deliv Rev 60: 876-885

89. Kratz F (2008) Albumin as a drug carrier: design of prodrugs, drug conjugates and nanoparticles. J Control Release 132: 171-183.

90. Stinchcombe TE (2007) Nanoparticle albumin-bound paclitaxel: a nove Cremphor-EL-free formulation of paclitaxel. Nanomedicine (Lond) 2: 415-423.

91. Karmali PP, Kotamraju VR, Kastantin M, Black M, Missirlis D, et al. (2009) Targeting of albumin-embedded paclitaxel nanoparticles to tumors. Nanomedicine 5: 73-82.

92. Zhao D, Zhao X, Zu Y, Li J, Zhang Y, et al. (2010) Preparation, characterization, and in vitro targeted delivery of folate-decorated paclitaxel-loaded bovine serum albumin nanoparticles. Int J Nanomedicine 5: 669-677.

93. Gong J, Huo M, Zhou J, Zhang Y, Peng X, et al. (2009) Synthesis characterization, drug-loading capacity and safety of novel octyl modified serum albumin micelles. Int J Pharm 376: 161-168.

94. Yadav AK, Mishra P, Agrawal GP (2008) An insight on hyaluronic acid in drug targeting and drug delivery. J Drug Target 16: 91-107.

95. Al-Ghananeem AM, Malkawi AH, Muammer YM, Balko JM, Black EP, et al. (2009) Intratumoral delivery of paclitaxel in solid tumor from biodegradable hyaluronan nanoparticle formulations. AAPS PharmSciTech 10: 410-417.

96. Saravanakumar G, Choi KY, Yoon HY, Kim K, Park JH, et al. (2010) Hydrotropic hyaluronic acid conjugates: synthesis, characterization, and implications as a carrier of paclitaxel. Int J Pharm 394: 154-161.

97. Salgueiro A, Gamisans F, Espina M, Alcober X, García ML, et al. (2002) Cyclophosphamide-loaded nanospheres: analysis of the matrix structure by thermal and spectroscopic methods. J Microencapsul 19: 305-310.

98. Huang CY, Chen CM, Lee YD (2007) Synthesis of high loading and encapsulation efficient paclitaxel-loaded poly (n-butyl cyanoacrylate) nanoparticles via miniemulsion. Int J Pharm 338: 267-275.

99. He M, Zhao Z, Yin L, Tang C, Yin C (2009) Hyaluronic acid coated poly(buty cyanoacrylate) nanoparticles as anticancer drug carriers. Int J Pharm 373: 165 173. 
100. Mitra A, Lin S (2003) Effect of surfactant on fabrication and characterization of paclitaxel-loaded polybutylcyanoacrylate nanoparticulate delivery systems. J Pharm Pharmacol 55: 895-902.

101. Kainthan RK, Muliawan EB, Hatzikiriakos SG, Brooks DE (2006) Synthesis, characterization, and viscoelastic properties of high molecular weight hyperbranched polyglycerols. Macromolecules 39: 7708-7717.

102. Mugabe C, Hadaschik BA, Kainthan RK, Brooks DE, So Al, et al. (2009) Paclitaxel incorporated in hydrophobically derivatized hyperbranched polyglycerols for intravesical bladder cancer therapy. BJU International 103 978-986.

103.Gao Z, Lukyanov AN, Singhal A, Torchilin VP (2002) Diacyllipid-polymer micelles as nanocarriers for poorly soluble anticancer drugs. Nano Letters 2 : 979-982.

104. Torchilin VP, Lukyanov AN, Gao Z, Papahadjopoulos-Sternberg B (2003) Immunomicelles: targeted pharmaceutical carriers for poorly soluble drugs. Proc Natl Acad Sci USA. 100: 6039-6044.

105. Wang J, Mongayt D, Torchilin VP (2005) Polymeric micelles for delivery of poorly soluble drugs: preparation and anticancer activity in vitro of paclitaxel incorporated into mixed micelles based on poly(ethylene glycol)-lipid conjugate and positively charged lipids. J Drug Target 13: 73-80.

106. Gao Z, Lukyanov AN, Chakilam AR, Torchilin VP (2003) PEG-PE/ phosphatidylcholine mixed immunomicelles specifically deliver encapsulated taxol to tumor cells of different origin and promote their efficient killing. J Drug Target 11: 87-92.

107. Krishnadas A, Rubinstein I, Onyüksel H (2003) Sterically stabilized phospholipid mixed micelles: in vitro evaluation as a novel carrier for waterinsoluble drugs. Pharm Res 20: 297-302.

108. Onyüksel H, Jeon E, Rubinstein I (2009) Nanomicellar paclitaxel increases cytotoxicity of multidrug resistant breast cancer cells. Cancer Lett 274: 327 330.

109. Lu Z, Yeh TK, Wang J, Chen L, Lyness G, et al. (2011) Paclitaxel gelatin nanoparticles for intravesical bladder cancer therapy. J Urol 185: 1478-1483.

110. Lu Z, Yeh TK, Tsai M, Au JL, Wientjes MG (2004) Paclitaxel-loaded gelatin nanoparticles for intravesical bladder cancer therapy. Clin Cancer Res 10: 7677-7684.

111. Yeh TK, Lu Z, Wientjes MG, Au JL (2005) Formulating paclitaxel in nanoparticles alters its disposition. Pharm Res 22: 867-874.

112. Tsai M, Lu Z, Wang J, Yeh TK, Wientjes MG, et al. (2007) Effects of carrier on disposition and antitumor activity of intraperitoneal paclitaxel. Pharm Res 24: 1691-1701.

113. Kataoka K, Kwon GS, Yokoyama M, Okano T, Sakurai Y (1993) Block copolymer micelles as vehicles for drug delivery. J Control Release 24: 119132.

114. Hamaguchi T, Matsumura Y, Suzuki M, Shimizu K, Goda R, et al. (2005) NK105, a paclitaxel-incorporating micellar nanoparticle formulation, can extend in vivo antitumour activity and reduce the neurotoxicity of paclitaxel. $\mathrm{Br}$ J Cancer 92: 1240-1246.

115. Hamaguchi T, Kato K, Yasui $\mathrm{H}$, Morizane $\mathrm{C}$, Ikeda M, et al. (2007) A phase $\mathrm{I}$ and pharmacokinetic study of NK105, a paclitaxel-incorporating micellar nanoparticle formulation. $\mathrm{Br} \mathrm{J}$ Cancer 97: 170-176.

116. Zhang JA, Anyarambhatla G, Ma L, Ugwu S, Xuan T, et al. (2005) Development and characterization of a novel Cremophor EL free liposome-based paclitaxel (LEP-ETU) formulation. Eur J Pharm Biopharm 59: 177-187.

117. Yoshizawa Y, Kono Y, Ogawara K, Kimura T, Higaki K (2011) PEG liposomalization of paclitaxel improved its in vivo disposition and anti-tumor efficacy. Int J Pharm 412: 132-141.

118. Fetterly GJ, Straubinger RM (2003) Pharmacokinetics of paclitaxel-containing liposomes in rats. AAPS PharmSci 5: E32: 90-100.

119. Bode C, Trojan L, Weiss C, Kraenzlin B, Michaelis U, et al. (2009) Paclitaxe encapsulated in cationic liposomes: a new option for neovascular targeting for the treatment of prostate cancer. Oncol Rep 22: 321-326.

120. Eichhorn ME, Ischenko I, Luedemann S, Strieth S, Papyan A, et al. (2010) Vascular targeting by EndoTAG ${ }^{\mathrm{TM}}-1$ enhances therapeutic efficacy of conventional chemotherapy in lung and pancreatic cancer. Int J Cancer 126 : $1235-1245$.
121. Serpe L, Catalano MG, Cavalli R, Ugazio E, Bosco O, et al. (2004) Cytotoxicity of anticancer drugs incorporated in solid lipid nanoparticles on HT-29 colorectal cancer cell line. Eur J Pharm Biopharm 58: 673-680.

122. Chen DB, Yang TZ, Lu WL, Zhang Q (2001) In vitro and in vivo study of two types of long-circulating solid lipid nanoparticles containing paclitaxel. Chem Pharm Bull (Tokyo) 49: 1444-1447

123. Lee MK, Lim SJ, Kim CK (2007) Preparation, characterization and in vitro cytotoxicity of paclitaxel-loaded sterically stabilized solid lipid nanoparticles. Biomaterials 28: 2137-2146.

124. Li R, Eun J, Lee MK (2011) Pharmacokinetics and biodistribution of paclitaxe loaded in pegylated solid lipid nanoparticles after intravenous administration. Archives Pharm Res 34: 331-337.

125. Pandita D, Ahuja A, Lather V, Benjamin B, Dutta T, et al. (2011) Development of lipid-based nanoparticles for enhancing the oral bioavailability of paclitaxel. AAPS PharmSciTech 12: 712-722.

126. Yuan H, Miao J, Du YZ, You J, Hu FQ, et al. (2008) Cellular uptake of solid lipid nanoparticles and cytotoxicity of encapsulated paclitaxel in A549 cance cells. Int J Pharm 348: 137-145.

127. Shenoy VS, Gude RP, Murthy RSR (2009) Paclitaxel-loaded glyceryl palmitostearate nanoparticles: in vitro release and cytotoxic activity. J Drug Target 17: 304-310.

128. Shenoy VS, Rajyaguru TH, Gude RP, Murthy RS (2009) Studies on paclitaxelloaded glyceryl monostearate nanoparticles. J Microencapsul 26: 471-478.

129. Arica-Yegin B, Benoît JP, Lamprecht A (2006) Paclitaxel-loaded lipid nanoparticles prepared by solvent injection or ultrasound emulsification. Drug Dev Ind Pharm 32: 1089-1094.

130. Koziara JM, Lockman PR, Allen DD, Mumper RJ (2004) Paclitaxe nanoparticles for the potential treatment of brain tumors. J Control Release 99: 259-269.

131. Koziara JM, Whisman TR, Tseng MT, Mumper RJ (2006) In-vivo efficacy o novel paclitaxel nanoparticles in paclitaxel-resistant human colorectal tumors. J Control Release 112: 312-319.

132. Dong X, Mattingly CA, Tseng MT, Cho S, Adams VR, et al. (2009) Development of new lipid-based paclitaxel nanoparticles using sequential simplex optimization. Eur J Pharm Biopharm 72: 9-17.

133. Dong X, Mattingly CA, Tseng MT, Cho MJ, Liu Y, et al. (2009) Doxorubicin and paclitaxel-loaded lipid-based nanoparticles overcome multidrug resistance by inhibiting P-glycoprotein and depleting ATP. Cancer Res 69: 3918-3926.

134. Hureaux J, Lagarce F, Gagnadoux F, Vecellio L, Roger E, et al. (2009) Lipid nanocapsules: ready-to-use nanovectors for the aerosol delivery of paclitaxel. Eur J Pharm Biopharm 73: 239-246.

135. Lacoeuille F, Hindre F, Moal F, Roux J, Passirani C, et al. (2007) In vivo evaluation of lipid nanocapsules as a promising colloidal carrier for paclitaxel. Int J Pharm 344: 143-149.

136. Vinchon-Petit S, Jarnet D, Paillard A, Benoit JP, Garcion E, et al. (2010) In vivo evaluation of intracellular drug-nanocarriers infused into intracranial tumours by convection-enhanced delivery: distribution and radiosensitisation efficacy. J Neurooncol 97: 195-205.

137. Hureaux J, Lagarce F, Gagnadoux F, Clavreul A, Benoit JP, et al. (2009) The adaptation of lipid nanocapsule formulations for blood administration in animals. Int. J. Pharm. 379: 266-269.

138. Hureaux J, Lagarce F, Gagnadoux F, Rousselet MC, Moal V, et al. (2010) Toxicological study and efficacy of blank and paclitaxel-loaded lipid nanocapsules after i.v. administration in mice. Pharm Res 27: 421-430.

139. Roger E, Lagarce F, Garcion E, Benoit JP (2010) Reciprocal competition between lipid nanocapsules and P-gp for paclitaxel transport across Caco-2 cells. Eur J Pharm Sci 40: 422-429.

140.Roger E, Lagarce F, Benoit JP (2009) The gastrointestinal stability of lipid nanocapsules. Int J Pharm 379: 260-265.

41. Ma P, Dong X, Swadley CL, Gupte A, Leggas M, et al. (2009) Development of idarubicin and doxorubicin solid lipid nanoparticles to overcome Pgp-mediated multiple drug resistance in leukemia. J Biomed Nanotechnol 5: 151-161.

142.Wang Z, Ho PC (2010) Self-assembled core-shell vascular-targeted nanocapsules for temporal antivasculature and anticancer activities. Small 6 : 2576-2583 
143. Wang Z, Ho PC (2010) A nanocapsular combinatorial sequential drug delivery system for antiangiogenesis and anticancer activities. Biomaterials 31: 71157123.

144.Bae KH, Lee Y, Park TG (2007) Oil-encapsulating PEO-PPO-PEO/PEG shell cross-linked nanocapsules for target-specific delivery of paclitaxel. Biomacromolecules 8: 650-656.

145. Jores K, Mehnert W, Drechsler M, Bunjes H, Johann C, et al. (2004) Investigations on the structure of solid lipid nanoparticles (SLN) and oilloaded solid lipid nanoparticles by photon correlation spectroscopy, field-flow fractionation and transmission electron microscopy. J Control Release 95: 217-227.

146. Ansell SM, Johnstone SA, Tardi PG, Lo L, Xie S, et al. (2008) Modulating the therapeutic activity of nanoparticle delivered paclitaxel by manipulating the hydrophobicity of prodrug conjugates. J Med Chem 51: 3288-3296.

147.Lundberg BB, Risovic V, Ramaswamy M, Wasan KM (2003) A lipophilic paclitaxel derivative incorporated in a lipid emulsion for parenteral administration. J Control Release 86: 93-100.

148. Perkins WR, Ahmad I, Li XG, Hirsh DJ, Masters GR, et al. (2000) Nove therapeutic nano-particles (lipocores): trapping poorly water soluble compounds. Int J Pharm 200: 27-39.

149. Stevens PJ, Sekido M, Lee RJ (2004) A folate receptor-targeted lipid nanoparticle formulation for a lipophilic paclitaxel prodrug. Pharm Res 21 2153-2157.

150.Ma P, Benhabbour RS, Feng L, Mumper RJ (2012) 2'-Behenoyl-paclitaxe conjugate containing lipid nanoparticles for the treatment of metastatic breast cancer. Cancer Lett (In Press).

151.Bedikian AY, DeConti RC, Conry R, Agarwala S, Papadopoulos N, et al (2011) Phase 3 study of docosahexaenoic acid-paclitaxel versus dacarbazine in patients with metastatic malignant melanoma. Ann Oncol 22: 787-793.

152. Bradley MO, Swindell CS, Anthony FH, Witman PA, Devanesan P, et al. (2001) Tumor targeting by conjugation of DHA to paclitaxel. J Control Release 74: 233-236.

153. Bradley MO, Webb NL, Anthony FH, Devanesan P, Witman PA, et al. (2001) Tumor targeting by covalent conjugation of a natural fatty acid to paclitaxel. Clin Cancer Res 7: 3229-3238.

154. Constantinides PP, Lambert KJ, Tustian AK, Schneider B, Lalji S, et al. (2000) Formulation development and antitumor activity of a filter-sterilizable emulsion of paclitaxel. Pharm Res 17: 175-182.

155. Bulitta JB, Zhao P, Arnold RD, Kessler DR, Daifuku R, et al. (2009) Mechanistic population pharmacokinetics of total and unbound paclitaxel for a new nanodroplet formulation versus Taxol in cancer patients. Cancer Chemother Pharmacol 63: 1049-1063.

156. Kan P, Chen ZB, Lee CJ, Chu IM (1999) Development of nonionic surfactant/ phospholipid o/w emulsion as a paclitaxel delivery system. J Control Release 58: 271-278.

157. Nornoo AO, Osborne DW, Chow DS (2008) Cremophor-free intravenous microemulsions for paclitaxel: I. formulation, cytotoxicity and hemolysis. Int $\mathrm{J}$ Pharm 349: 108-116.

158. Nornoo AO, Chow DS (2008) Cremophor-free intravenous microemulsions for paclitaxel: II. stability, in vitro release and pharmacokinetics. Int J Pharm 349: 117-123.

159. Tiwari SB, Amiji MM (2006) Improved oral delivery of paclitaxel following administration in nanoemulsion formulations. J Nanosci Nanotechnol 6: 32153221.

160.Ganta S, Amiji M (2009) Coadministration of Paclitaxel and curcumin in nanoemulsion formulations to overcome multidrug resistance in tumor cells. Mol Pharm 6: 928-939.

161. Khandare JJ, Jayant S, Singh A, Chandna P, Wang Y, et al. (2006) Dendrimer versus linear conjugate: influence of polymeric architecture on the delivery and anticancer effect of paclitaxel. Bioconjug Chem 17: 1464-1472.

162. Majoros IJ, Myc A, Thomas T, Mehta CB, Baker JR Jr (2006) PAMAM dendrimer-based multifunctional conjugate for cancer therapy: synthesis, characterization, and functionality. Biomacromolecules 7: 572-579.

163. Wang Y, Xin D, Liu K, Zhu M, Xiang J (2009) Heparin-paclitaxel conjugates as drug delivery system: synthesis, self-assembly property, drug release, and antitumor activity. Bioconjug Chem 20: 2214-2221.
164. Xin D, Wang Y, Xiang J (2010) The use of amino acid linkers in the conjugation of paclitaxel with hyaluronic acid as drug delivery system: synthesis, selfassembled property, drug release, and in vitro efficiency. Pharm Res 27: 380389.

165.Wang X, Li J, Wang Y, Cho KJ, Kim G, et al. (2009) HFT-T, a targeting nanoparticle, enhances specific delivery of paclitaxel to folate receptorpositive tumors. ACS Nano 3: 3165-3174

166. Yang D, Van S, Jiang X, Yu L (2011) Novel free paclitaxel-loaded poly(LY-glutamylglutamine)-paclitaxel nanoparticles. Int J Nanomedicine 6: 85-91.

167. Feng Z, Zhao G, Yu L, Gough D, Howell SB (2010) Preclinical efficacy studies of a novel nanoparticle-based formulation of paclitaxel that out-performs Abraxane. Cancer Chemother Pharmacol 65: 923-930.

168. Van S, Das SK, Wang X, Feng Z, Jin Y, et al. (2010) Synthesis, characterization, and biological evaluation of poly(L-y -glutamyl-glutamine $)$ paclitaxel nanoconjugate. Int J Nanomedicine 5: 825-837.

169.Peng L, Das K, Yu L, Howell S, Gough D (2011) Coarse-grained modeling study of nonpeptide RGD ligand density and PEG molecular weight on the conformation of poly(y-glutamyl-glutamate) paclitaxel conjugates. J Mol Model 17: 2973-87.

170. Meerum Terwogt JM, ten Bokkel Huinink WW, Schellens JH, Schot M, Mandjes IA, et al. (2001) Phase I clinical and pharmacokinetic study of PNU166945 a novel water-soluble polymer-conjugated prodrug of paclitaxel. Anticancer Drugs 12: 315-323.

171. Galic VL, Herzog TJ, Wright JD, Lewin SN (2011) Paclitaxel poliglumex for ovarian cancer. Expert Opin Investig Drugs 20: 813-821.

172.Singer JW (2005) Paclitaxel poliglumex (XYOTAX, CT-2103): a macromolecular taxane. J Control Release 109: 120-126.

173. Gibson JD, Khanal BP, Zubarev ER (2007) Paclitaxel-functionalized gold nanoparticles. J Am Chem Soc 129: 11653-11661.

174. Oh KS, Kim RS, Lee J, Kim D, Cho SH, et al. (2008) Gold/chitosan/pluronic composite nanoparticles for drug delivery. J Appl Polym Sci 108(5):3239-3244.

175. Heo DN, Yang DH, Moon HJ, Lee JB, Bae MS, et al. (2012) Gold nanoparticles surface-functionalized with paclitaxel drug and biotin receptor as theranostic agents for cancer therapy. Biomaterials 33: 856-866.

176. Hua MY, Yang HW, Chuang CK, Tsai RY, Chen WJ, et al. (2010) Magneticnanoparticle-modified paclitaxel for targeted therapy for prostate cancer. Biomaterials 31: 7355-7363.

177. Hwu JR, Lin YS, Josephrajan T, Hsu MH, Cheng FY, et al. (2009) Targeted paclitaxel by conjugation to iron oxide and gold nanoparticles. J Am Chem Soc 131: 66-68.

178. Liu Z, Chen K, Davis C, Sherlock S, Cao Q, et al. (2008) Drug delivery with carbon nanotubes for in vivo cancer treatment. Cancer Res 68: 6652-6660.

179. Lay CL, Liu HQ, Tan HR, Liu Y (2010) Delivery of paclitaxel by physically loading onto poly(ethylene glycol) (PEG)-graft-carbon nanotubes for potent cancer therapeutics. Nanotechnology 21: 065101

180. Sobhani Z, Dinarvand R, Atyabi F, Ghahremani M, Adeli M (2011) Increased paclitaxel cytotoxicity against cancer cell lines using a novel functionalized carbon nanotube. Int J Nanomedicine 6: 705-719.

181. Liu Y, Huang L, Liu F (2010) Paclitaxel nanocrystals for overcoming multidrug resistance in cancer. Mol Pharm 7: 863-869.

182. Liu F, Park JY, Zhang Y, Conwell C, Liu Y, et al. (2010) Targeted cancer therapy with novel high drug-loading nanocrystals. J Pharm Sci 99: 35423551.

183. Deng J, Huang L, Liu F (2010) Understanding the structure and stability of paclitaxel nanocrystals. Int J Pharm 390: 242-249.

184.Zhang X, Zhang X, Wu Z, Gao X, Cheng C, et al. (2011) A hydrotropic $\beta$-cyclodextrin grafted hyperbranched polyglycerol co-polymer for hydrophobic drug delivery. Acta Biomater 7: 585-592.

185. Bilensoy E, Gürkaynak O, Ertan M, Sen M, Hincal AA (2008) Development of nonsurfactant cyclodextrin nanoparticles loaded with anticancer drug paclitaxel. J Pharm Sci 97: 1519-1529.

186. Bilensoy E, Gürkaynak O, Doğan AL, Hincal AA (2008) Safety and efficacy of amphiphilic beta-cyclodextrin nanoparticles for paclitaxel delivery. Int J Pharm 347: 163-170. 
Citation: Ma P, Mumper RJ (2013) Paclitaxel Nano-Delivery Systems: A Comprehensive Review. J Nanomed Nanotechol 4: 164. doi:10.4172/21577439.1000164

Page 16 of 16

187. Memisoglu E, Bochot A, Sen M, Charon D, Duchêne D, et al. (2002) Amphiphilic beta-cyclodextrins modified on the primary face: synthesis, characterization, and evaluation of their potential as novel excipients in the preparation of nanocapsules. J Pharm Sci 91: 1214-1224.

188. Lee JY, Kim KS, Kang YM, Kim ES, Hwang SJ, et al. (2010) In vivo efficacy of paclitaxel-loaded injectable in situ-forming gel against subcutaneous tumor growth. Int J Pharm 392: 51-56.

189. Jeong B, Bae YH, Lee DS, Kim SW (1997) Biodegradable block copolymers as injectable drug-delivery systems. Nature 388: 860-862.

190.Zentner GM, Rathi R, Shih C, McRea JC, Seo MH, et al. (2001) Biodegradable block copolymers for delivery of proteins and water-insoluble drugs. J Control Release 72: 203-215.
191. Wen PY (2010) American Society of Clinical Oncology 2010: report of selected studies from the CNS tumors section. Expert Rev Anticancer Ther 10: 13671369.

192. Regina A, Demeule M, Che C, Lavallee I, Poirier J, et al. (2008) Antitumour activity of ANG1005, a conjugate between paclitaxel and the new brain delivery vector Angiopep-2. Br J Pharmacol 155: 185-197.

193. Mei L, Sun H, Song C (2009) Local delivery of modified paclitaxel-loaded poly(epsilon-caprolactone)/pluronic F68 nanoparticles for long-term inhibition of hyperplasia. J Pharm Sci 98: 2040-2050.

194. Kim JH, Kim YS, Kim S, Park JH, Kim K, et al. (2006) Hydrophobically modified glycol chitosan nanoparticles as carriers for paclitaxel. J Control Release 111 228-234. 